\title{
Rank 2 Nichols Algebras of Diagonal Type over Fields of Positive Characteristic
}

\author{
Jing WANG and István HECKENBERGER
}

Philipps-Universität Marburg, FB Mathematik und Informatik, Hans-Meerwein-Straße, 35032 Marburg, Germany

E-mail: jing@mathematik.uni-marburg.de, heckenberger@mathematik.uni-marburg.de

URL: http://www.mathematik.uni-marburg.de/ jing/, http://www. mathematik. uni-marburg.de/ heckenberger/

Received August 01, 2014, in final form February 02, 2015; Published online February 07, 2015 http://dx.doi.org/10.3842/SIGMA.2015.011

\begin{abstract}
The paper introduces a new method to determine all rank two Nichols algebras of diagonal type over fields of positive characteristic.
\end{abstract}

Key words: Nichols algebra; Cartan graph; Weyl groupoid; root system

2010 Mathematics Subject Classification: 16T05

\section{Introduction}

The theory of Nichols algebras was dominated and motivated by Hopf algebra theory. In 1978, W. Nichols first introduced the structure of Nichols algebra in the paper "Bialgebras of type one" [22], where he studied certain pointed Hopf algebras. S.L. Woronowicz rediscovered this structure in his approach to "quantum differential calculus" [28, 29]. M. Rosso and G. Lusztig defined and used them to present quantum groups in a different language [21, 23]. In fact, Nichols algebra turns out to be very important in Hopf algebras and quantum groups [2, 6, 24] and has applications in conformal field theory and mathematical physics [25, 26, 27]. Nichols algebras play an important role in the classification of pointed Hopf algebras with certain finiteness properties in papers by N. Andruskiewitsch and H.-J. Schneider, see for example [4, 6]. The crucial step to classify pointed Hopf algebras is the computation of the Nichols algebras. The explicit presentations by generators and relations of a finite-dimensional Nichols algebra of a braided vector space in suitable classes are crucial for the classification lifting method in $[4,6]$.

Several authors have already classified both infinite and finite dimensional Nichols algebra of Cartan type, see [5, 12, 23]. Further, N. Andruskiewitsch [1] stated the following question.

Question 5.9. Given a braiding matrix $\left(q_{i j}\right)_{1 \leq i, j \leq \theta}$ whose entries are roots of 1 , when $\mathcal{B}(V)$ is finite-dimensional, where $V$ is a vector space with basis $x_{1}, \ldots, x_{\theta}$ and braiding $c\left(x_{i} \otimes x_{j}\right)=$ $q_{i j}\left(x_{j} \otimes x_{i}\right)$ ? If so, compute $\operatorname{dim}_{\mathbb{k}} \mathcal{B}(V)$, and give a "nice" presentation by generators and relations.

The first half of Question 5.9 was answered by the first named author in [14] when the characteristic of the field is 0 . The crucial theoretical tools of the classification were the Weyl groupoid of a braided vector space of diagonal type and the root system associated to a Nichols algebra of diagonal type, see [12]. From V. Kharchenko [20, Theorem 2] any Nichols algebra $\mathcal{B}(V)$ of diagonal type has a (restricted) Poincaré-Birkhoff-Witt basis consisting of homogenous elements with respect to the $\mathbb{Z}^{n}$-grading of $\mathcal{B}(V)$. In [12], the root system and the Weyl groupoid of $\mathcal{B}(V)$ for a Nichols algebra $\mathcal{B}(V)$ of diagonal type was defined. This Weyl groupoid plays a similar role as the Weyl group does for ordinary root systems. Based on these results, in [18] and [10] the abstract combinatorial theory of Weyl groupoids and generalized root systems was initiated. 
Later, the theory of root systems and Weyl groupoids was extended to more general Nichols algebras in $[3,16,17]$. With the classification result in [14], N. Andruskiewitsch and H.-J. Schneider [7] obtained a classification theorem about finite-dimensional pointed Hopf algebras under some technical assumptions. On the other hand, it is natural and desirable to analyze the classification of Nichols algebras of diagonal type for arbitrary fields. The authors in [8] constructed new examples of Nichols algebras in positive characteristic by applying a combinatorial formula for products in Hopf quiver algebras.

In this paper, all rank 2 Nichols algebras of diagonal type with a finite root system over fields of positive characteristic are classified. We introduce some properties of rank two Cartan graphs, see Theorem 4.15. Theorem 4.15 characterizes finite connected Cartan graphs of rank two in terms of certain integer sequences. This theorem simplifies substantially the calculations needed to check that the Weyl groupoids of the Nichols algebras in Tables 5.1-5.6 of our classification are finite. Indeed, using the theorem, these calculations can be done by hand within a very short time, in contrast to the calculations based on the definition of the Weyl groupoid $\mathcal{W}$ or using a longest element of $\mathcal{W}$. The main result of this paper is Theorem 5.1. Table 7 illustrates all the exchange graphs of the corresponding Cartan graphs in Theorem 5.1.

The structure of the paper is as follows. In Section 2 we recall the definition of Cartan graphs, their Weyl groupoids and root systems. Some well-known results are also recalled. In Section 3, Theorem 3.1 associates a semi-Cartan graph $\mathcal{C}(M)$ of rank $\theta$ to a tuple $M$ of finite-dimensional irreducible Yetter-Drinfel'd-modules. Further, $\mathcal{C}(M)$ is a Cartan graph if the set of real roots of $M$ is finite. The Dynkin diagram for a braided vector space of diagonal type is recalled in Section 3.1 and some corollaries are also obtained, see Lemma 3.4 and Proposition 3.6. In Section 4 we recall certain integer sequences and prove a local property of them in Theorem 4.6. This is another main technical part for the classification. Finally the main result of this paper is formulated in Section 5, see Theorem 5.1. In this section, all rank two Nichols algebras of diagonal type with a finite root system over fields of positive characteristic are classified. Since many subcases have to be considered, this is the largest part of the paper. To simplify the results, this paper ends with 5 tables containing all the Dynkin diagrams of rank two braided vector spaces with a finite root system for all fields of positive characteristic.

Throughout the paper $\mathbb{k}$ denotes a field of characteristic $p>0$. Let $\mathbb{k}^{*}=\mathbb{k} \backslash\{0\}$. The set of natural numbers not including 0 is denoted by $\mathbb{N}$ and we write $\mathbb{N}_{0}=\mathbb{N} \cup\{0\}$. For $n \in \mathbb{N}$, let $G_{n}^{\prime}$ denote the set of primitive $n$-th roots of unity in $\mathbb{k}$, that is $G_{n}^{\prime}=\left\{q \in \mathbb{k}^{*} \mid q^{n}=1\right.$, $q^{k} \neq 1$ for all $\left.1 \leq k<n\right\}$.

\section{Cartan graphs and root systems}

Let $I$ be a non-empty finite set. Recall from $[19, \S 1.1]$ that a generalized Cartan matrix is a matrix $A=\left(a_{i j}\right)_{i, j \in I}$ with integer entries such that

- $a_{i i}=2$ and $a_{j k} \leq 0$ for any $i, j, k \in I$ with $j \neq k$,

- if $a_{i j}=0$ for some $i, j \in I$, then $a_{j i}=0$.

Let $\mathcal{X}$ be a non-empty set and let $r: I \times \mathcal{X} \rightarrow \mathcal{X}$ be a map. For all $i \in I$, let $r_{i}: \mathcal{X} \rightarrow \mathcal{X}$, $X \mapsto r(i, X)$. Let $A^{X}=\left(a_{i j}^{X}\right)_{i, j \in I}$ be a generalized Cartan matrix in $\mathbb{Z}^{I \times I}$ for all $X \in \mathcal{X}$. The quadruple $\mathcal{C}=\mathcal{C}\left(I, \mathcal{X}, r,\left(A^{X}\right)_{X \in \mathcal{X}}\right)$ is called a semi-Cartan graph if

- $r_{i}^{2}=\operatorname{id}_{\mathcal{X}}$ for all $i \in I$,

- $a_{i j}^{X}=a_{i j}^{r_{i}(X)}$ for all $X \in \mathcal{X}$ and $i, j \in I$.

The cardinality of $I$ is called the rank of $\mathcal{C}$, and the elements of $I$ the labels of $\mathcal{C}$. The elements of $\mathcal{X}$ are called the points of $\mathcal{C}$. Semi-Cartan graphs are called Cartan schemes in [9]. 
We change the terminology in order to increase the recognizability of our structures, to avoid possible confusion with other mathematical concepts, and in order to shorten the notation for our main objects, the Cartan graphs. We thank N. Andruskiewitsch and H.-J. Schneider for a very fruitful discussion on this issue.

The exchange graph of $\mathcal{C}$ is a labeled non-oriented graph with vertices corresponding to points of $\mathcal{C}$, and edges marked by labels of $\mathcal{C}$, where two vertices $X, Y$ are connected by an edge $i$ if and only if $X \neq Y$ and $r_{i}(X)=Y$ (and $r_{i}(Y)=X$ ). For simplification, instead of several edges we display only one edge with several labels. A semi-Cartan graph is called connected if its exchange graph is a connected graph.

Let $\mathcal{C}=\mathcal{C}\left(I, \mathcal{X}, r,\left(A^{X}\right)_{X \in \mathcal{X}}\right)$ be a semi-Cartan graph. We fix once and for all the notation $\left(\alpha_{i}\right)_{i \in I}$ for the standard basis of $\mathbb{Z}^{I}$. Then there exists a unique category $\mathcal{D}(\mathcal{X}, I)$ with objects $\operatorname{ObD}(\mathcal{X}, I)=\mathcal{X}$ and morphisms $\operatorname{Hom}(X, Y)=\left\{(Y, f, X) \mid f \in \operatorname{End}\left(\mathbb{Z}^{I}\right)\right\}$ for $X, Y \in \mathcal{X}$, such that the composition is defined by

$$
(Z, g, Y) \circ(Y, f, X)=(Z, g f, X)
$$

for all $X, Y, Z \in \mathcal{X}, f, g \in \operatorname{End}\left(\mathbb{Z}^{I}\right)$. For all $X \in \mathcal{X}$ and all $i \in I$, let

$$
s_{i}^{X} \in \operatorname{Aut}\left(\mathbb{Z}^{I}\right), \quad s_{i}^{X} \alpha_{j}=\alpha_{j}-a_{i j}^{X} \alpha_{i}
$$

for all $j \in I$. We write $\mathcal{W}(\mathcal{C})$ for the smallest subcategory of $\mathcal{D}(\mathcal{X}, I)$ which contains all morphisms $\left(r_{i}(X), s_{i}^{X}, X\right)$, where $i \in I$ and $X \in \mathcal{X}$. The morphisms $\left(r_{i}(X), s_{i}^{X}, X\right)$ are usually abbreviated by $s_{i}^{X}$, or by $s_{i}$, if no confusion is possible. Since all generators are invertible $\mathcal{W}(\mathcal{C})$ is a groupoid.

Let $\mathcal{C}=\mathcal{C}\left(I, \mathcal{X}, r,\left(A^{X}\right)_{X \in \mathcal{X}}\right)$ be a semi-Cartan graph and $\left(\boldsymbol{\Delta}^{X}\right)_{X \in \mathcal{X}}$ a family of sets $\boldsymbol{\Delta}^{X} \subset \mathbb{Z}^{I}$. We say that $\mathcal{R}=\mathcal{R}\left(\mathcal{C},\left(\boldsymbol{\Delta}^{X}\right)_{X \in \mathcal{X}}\right)$ is a root system of type $\mathcal{C}$ if and only if

- $\boldsymbol{\Delta}^{X}=\left(\boldsymbol{\Delta}^{X} \cap \mathbb{N}_{0}^{I}\right) \cup-\left(\boldsymbol{\Delta}^{X} \cap \mathbb{N}_{0}^{I}\right)$,

- $\boldsymbol{\Delta}^{X} \cap \mathbb{Z} \alpha_{i}=\left\{\alpha_{i},-\alpha_{i}\right\}$ for all $i \in I$,

- $s_{i}^{X}\left(\boldsymbol{\Delta}^{X}\right)=\boldsymbol{\Delta}^{r_{i}(X)}$ for all $i \in I$,

- $\left(r_{i} r_{j}\right)^{m_{i j}^{X}}(X)=X$ for any $i, j \in I$ such that $i \neq j$ where $m_{i j}^{X}:=\left|\Delta^{X} \cap\left(\mathbb{N}_{0} \alpha_{i}+\mathbb{N}_{0} \alpha_{j}\right)\right|$ is finite.

For any category $\mathcal{D}$ and any object $X$ in $\mathcal{D}$, let $\operatorname{Hom}(\mathcal{D}, X)=\cup_{Y \in \mathcal{D}} \operatorname{Hom}(Y, X)$. For all $X \in \mathcal{X}$, the set

$$
\boldsymbol{\Delta}^{X \mathrm{re}}=\left\{w \alpha_{i} \in \mathbb{Z}^{I} \mid w \in \operatorname{Hom}(\mathcal{W}(\mathcal{C}), X)\right\}
$$

is called the set of real roots of $\mathcal{C}$ at $X$. The elements of $\Delta_{+}^{X \mathrm{re}}=\boldsymbol{\Delta}^{X \mathrm{re}} \cap \mathbb{N}_{0}^{I}$ are called positive roots and those of $\boldsymbol{\Delta}^{X \mathrm{re}} \cap-\mathbb{N}_{0}^{I}$ negative roots. A semi-Cartan graph is called finite if $\boldsymbol{\Delta}^{X \mathrm{re}}$ is a finite set for all $X \in \mathcal{X}$.

Let $t_{i j}^{X}=\left|\boldsymbol{\Delta}^{X \text { re }} \cap\left(\mathbb{N}_{0} \alpha_{i}+\mathbb{N}_{0} \alpha_{j}\right)\right|$. We say that $\mathcal{C}$ is a Cartan graph if the following hold:

- For all $X \in \mathcal{X}$ the set $\boldsymbol{\Delta}^{X \text { re }}$ consists of positive and negative roots.

- Let $X \in \mathcal{X}$ and $i, j \in I$. If $t_{i j}^{X}<\infty$ then $\left(r_{i} r_{j}\right)^{t_{i j}^{X}}(X)=X$.

In that case, $\mathcal{W}(\mathcal{C})$ is called the Weyl groupoid of $\mathcal{C}$.

\section{Remark 2.1.}

- A semi-Cartan graph $\mathcal{C}$ is a Cartan graph if and only if $\mathcal{R}=\mathcal{R}\left(\mathcal{C},\left(\boldsymbol{\Delta}^{X \mathrm{re}}\right)_{X \in \mathcal{X}}\right)$ is a root system of type $\mathcal{C}$.

- For any finite Cartan graph $\mathcal{C}$, there is a unique root system $\mathcal{R}=\mathcal{R}\left(\mathcal{C},\left(\boldsymbol{\Delta}^{X \mathrm{re}}\right)_{X \in \mathcal{X}}\right)$ of type $\mathcal{C}$, see [10, Propositions 2.9, 2.12]. 


\section{Cartan graphs for Nichols algebras}

Let $H$ be a Hopf algebra over $\mathbb{k}$ with bijective antipode. Let ${ }_{H}^{H} \mathcal{Y} \mathcal{D}$ denote the category of Yetter-Drinfel'd modules over $H$ and $\mathcal{F}_{\theta}^{H}$ the set of $\theta$-tuples of finite-dimensional irreducible objects in ${ }_{H}^{H} \mathcal{Y} \mathcal{D}$ for all $\theta \in \mathbb{N}$. For all $V \in{ }_{H}^{H} \mathcal{Y D}$, let $\delta$ denote the left coaction of $H$ on $V$ and the left action of $H$ on $V$. Let $\theta \in \mathbb{N}, I=\{1, \ldots, \theta\}$, and $M=\left(M_{1}, \ldots, M_{\theta}\right) \in \mathcal{F}_{\theta}^{H}$. Write $[M]=\left(\left[M_{1}\right], \ldots,\left[M_{\theta}\right]\right) \in \mathcal{X}_{\theta}^{H}$, where $\mathcal{X}_{\theta}^{H}$ denotes the set of $\theta$-tuples of isomorphism classes of finite-dimensional irreducible objects in ${ }_{H}^{H} \mathcal{Y} \mathcal{D}$. Let $\mathcal{B}(M)$ denote the Nichols algebra $\mathcal{B}\left(M_{1} \oplus \cdots \oplus M_{\theta}\right)$. The Nichols algebra $\mathcal{B}(V)$ is known to be a $\mathbb{N}_{0}^{\theta}$-graded algebra and coalgebra in ${ }_{H}^{H} \mathcal{Y} \mathcal{D}$ such that $\operatorname{deg} M_{i}=\alpha_{i}$ for all $i \in I$.

The adjoint action in the braided category ${ }_{H}^{H} \mathcal{Y D}$ is given by $\operatorname{ad}_{c} x(y)=x y-\left(x_{(-1)} \cdot y\right) x_{(0)}$ for all $x \in M_{1} \oplus \cdots \oplus M_{\theta}, y \in \mathcal{B}(M)$, where $\delta(x)=x_{(-1)} \otimes x_{(0)}$.

By [17, Definition 6.8], the Nichols algebra $\mathcal{B}(M)$ is called decomposable if there exists a totally ordered index set $(L, \leq)$ and a family $\left(W_{l}\right)_{l \in L}$ of finite-dimensional irreducible $\mathbb{N}_{0}^{\theta}$-graded objects in ${ }_{H}^{H} \mathcal{Y D}$ such that

$$
\mathcal{B}(M) \simeq \bigotimes_{l \in L} \mathcal{B}\left(W_{l}\right)
$$

Decomposability of $\mathcal{B}(M)$ is known under several assumptions on $M$. In particular, if $H$ is a group algebra of an abelian group and $\operatorname{dim} M_{i}=1$ for all $1 \leq i \leq \theta$, then $\mathcal{B}(M)$ is decomposable by a theorem of V. Kharchenko [20, Theorem 2].

Assume that $\mathcal{B}(M)$ is decomposable. One defines for any decomposition (3.1) the set of positive roots $\boldsymbol{\Delta}_{+}^{[M]} \subset \mathbb{Z}^{I}$ and the set of roots $\boldsymbol{\Delta}^{[M]} \subset \mathbb{Z}^{I}$ of $[M]$ by

$$
\boldsymbol{\Delta}_{+}^{[M]}=\left\{\operatorname{deg}\left(W_{l}\right) \mid l \in L\right\}, \quad \boldsymbol{\Delta}^{[M]}=\boldsymbol{\Delta}_{+}^{[M]} \cup-\boldsymbol{\Delta}_{+}^{[M]} .
$$

The set of roots of $[M]$ does not depend on the choice of the decomposition (3.1).

Let $i \in I$. Following [16, Definition 6.4] we say that $M$ is $i$-finite, if for any $j \in I \backslash\{i\}$, $\left(\operatorname{ad}_{c} M_{i}\right)^{m}\left(M_{j}\right)=0$ for some $m \in \mathbb{N}$. Assume that $M$ is $i$-finite. Let $\left(a_{i j}^{M}\right)_{j \in I} \in \mathbb{Z}^{I}$ and $R_{i}(M)=\left(R_{i}(M)_{j}\right)_{j \in I}$, where

$$
\begin{aligned}
& a_{i j}^{M}=\left\{\begin{array}{lll}
2 & \text { if } & j=i, \\
-\max \left\{m \in \mathbb{N}_{0} \mid\left(\operatorname{ad}_{c} M_{i}\right)^{m}\left(M_{j}\right) \neq 0\right\} & \text { if } & j \neq i,
\end{array}\right. \\
& R_{i}(M)_{i}=M_{i}^{*}, \quad R_{i}(M)_{j}=\left(\operatorname{ad}_{c} M_{i}\right)^{-a_{i j}^{M}}\left(M_{j}\right) \text {. }
\end{aligned}
$$

Then $R_{i}(M)_{j}$ is irreducible by [16, Theorem 7.2(3)]. If $M$ is not $i$-finite, then let $R_{i}(M)=M$. Let

$$
\begin{aligned}
& \mathcal{X}_{\theta}^{H}(M)=\left\{\left[R_{i_{1}} \cdots R_{i_{n}}(M)\right] \in \mathcal{X}_{\theta}^{H} \mid n \in \mathbb{N}_{0}, i_{1}, \ldots, i_{n} \in I\right\}, \\
& \mathcal{F}_{\theta}^{H}(M)=\left\{R_{i_{1}} \cdots R_{i_{n}}(M) \in \mathcal{F}_{\theta}^{H} \mid n \in \mathbb{N}_{0}, i_{1}, \ldots, i_{n} \in I\right\} .
\end{aligned}
$$

We say that $M \in \mathcal{F}_{\theta}^{H}$ admits all reflections if $N$ is $i$-finite for all $N \in \mathcal{F}_{\theta}^{H}(M)$.

Theorem 3.1. Let $M \in \mathcal{F}_{\theta}^{H}$. Assume that $M$ admits all reflections. Let $r: I \times \mathcal{X}_{\theta}^{H}(M) \rightarrow$ $\mathcal{X}_{\theta}^{H}(M),(i,[N]) \mapsto\left[R_{i}(N)\right]$ for all $i \in I$. Then

$$
\mathcal{C}(M)=\left\{I, \mathcal{X}_{\theta}^{H}(M), r,\left(A^{[N]}\right)_{[N] \in \mathcal{X}_{\theta}^{H}(M)}\right\}
$$

is a semi-Cartan graph. If moreover $\boldsymbol{\Delta}^{[M] \mathrm{re}}$ is finite, then $\mathcal{C}(M)$ is a Cartan graph. 
Proof. The first claim follows from [3, Theorem 3.12], see [16, Theorem 6.10] for details. If $\boldsymbol{\Delta}^{[M] \mathrm{re}}$ is finite and $M$ admits all reflections, then

$$
\mathcal{R}(M)=\mathcal{R}\left(\mathcal{C}(M),\left(\Delta^{X \mathrm{re}}\right)_{X \in \mathcal{X}_{\theta}^{H}(M)}\right)
$$

is a root system of type $\mathcal{C}(M)$ by [17, Corollary 6.16]. Hence $\mathcal{C}(M)$ is a Cartan graph because of [10, Proposition 2.9].

Therefore if $M$ admits all reflections then we can attach the groupoid $\mathcal{W}(M):=\mathcal{W}(\mathcal{C}(M))$ to $M$.

\subsection{Small Cartan graphs for Nichols algebras of diagonal type}

Let $G$ be an abelian group and let $V$ be a Yetter-Drinfel'd module over $\mathbb{k} G$ of rank $\theta$ with a basis $\left\{x_{i} \mid i \in I\right\}$. Let.$: \mathbb{k} G \otimes V \rightarrow V$ and $\delta: V \rightarrow \mathbb{k} G \otimes V$ denote the left action and the left coaction of $\mathbb{k} G$ on $V$, respectively. Assume that $V$ is of diagonal type. More precisely, let $\left\{g_{i} \mid i \in I\right\}$ be a subset of $G$ and $q_{i j} \in \mathbb{k}^{*}$ for all $i, j \in I$, such that

$$
\delta\left(x_{i}\right)=g_{i} \otimes x_{i}, \quad g_{i} . x_{j}=q_{i j} x_{j}
$$

for $i, j \in I$. Then $V$ is a braided vector space of dimension $\theta$ [1, Definition 5.4] and the braiding $c \in \operatorname{End}(V \otimes V)$ is of diagonal type, that is $c\left(x_{i} \otimes x_{j}\right)=q_{i j} x_{j} \otimes x_{i}$ for all $i, j \in I$. Then $\left(q_{i j}\right)_{i, j \in I}$ is the braiding matrix of $V$ with respect to the basis $\left\{x_{i} \mid i \in I\right\}$.

The braiding matrix is known to be independent of the basis $\left\{x_{i} \mid i \in I\right\}$, up to permutation of $I$. It can be obtained for example from [15, Proposition 1.3] using the arguments in its proof.

The Nichols algebra $\mathcal{B}(V)$ generated by $V$ is said to be of diagonal type [1, Definition 5.8].

For $\rho \in \mathcal{B}(V)$, the braided commutator $\operatorname{ad}_{c}$ takes the form $\operatorname{ad}_{c} x_{i}(\rho)=x_{i} \rho-\left(g_{i} . \rho\right) x_{i}$.

Lemma 3.2. Let $M=\left(M_{i}\right)_{i \in I} \in \mathcal{F}_{\theta}^{\mathbb{k} G}$ be a tuple of one-dimensional Yetter-Drinfel'd modules over $\mathbb{k} G$ and let $x_{i}$ be a basis of $M_{i}$, for all $i \in I$. Let $m \in \mathbb{N}_{0}$. For any $i, j \in I$ with $i \neq j$, the following are equivalent:

(a) $(m+1)_{q_{i i}}\left(q_{i i}^{m} q_{i j} q_{j i}-1\right)=0$ and $(k+1)_{q_{i i}}\left(q_{i i}^{k} q_{i j} q_{j i}-1\right) \neq 0$ for all $0 \leq k<m$,

(b) $\left(\operatorname{ad}_{c} x_{i}\right)^{m+1}\left(x_{j}\right)=0$ and $\left(\operatorname{ad}_{c} x_{i}\right)^{m}\left(x_{j}\right) \neq 0$ in $\mathcal{B}(V)$,

(c) $-a_{i j}^{M}=m$.

Here $(n)_{q}:=1+q+\cdots+q^{n-1}$, which is 0 if and only if $q^{n}=0$ for $q \neq 1$ or $p \mid n$ for $q=1$.

Proof. $(\mathrm{a}) \Leftrightarrow(\mathrm{b})$ follows from [6, Lemma 3.7] and (b) $\Leftrightarrow(\mathrm{c})$ holds by the definition of $a_{i j}^{M}$.

Lemma 3.3. Let $i \in I$. Then $M=\left(M_{j}\right)_{j \in I} \in \mathcal{F}_{\theta}^{\mathbb{k} G}$ is $i$-finite if and only if for any $j \in I \backslash\{i\}$ there is a non-negative integer $m$ satisfying $(m+1) q_{i i}\left(q_{i i}^{m} q_{i j} q_{j i}-1\right)=0$.

Proof. The claim follows from Lemma 3.2.

Let $V$ be a $\theta$-dimensional braided vector space of diagonal type. Let $\left(q_{i j}\right)_{i, j \in I}$ be a braiding matrix of $V$. The Dynkin diagram [13, Definition 4] of $V$ is denoted by $\mathcal{D}$. It is a non-directed graph with the following properties:

- there is a bijective map $\phi$ from $I$ to the vertices of $\mathcal{D}$,

- for all $i \in I$ the vertex $\phi(i)$ is labeled by $q_{i i}$,

- for any $i, j \in I$ with $i \neq j$, the number $n_{i j}$ of edges between $\phi(i)$ and $\phi(j)$ is either 0 or 1 . If $q_{i j} q_{j i}=1$ then $n_{i j}=0$, otherwise $n_{i j}=1$ and the edge is labeled by $q_{i j} q_{j i}$. 
Let $M=\left(M_{i}\right)_{i \in I} \in \mathcal{F}_{\theta}^{\mathbb{k} G}$ be a tuple of one-dimensional Yetter-Drinfel'd modules over $\mathbb{k} G$. The Dynkin diagram of $M$ is the Dynkin diagram of the braided vector space $M_{1} \oplus \cdots \oplus M_{\theta}$.

Let $i \in I$. Assume that $M$ is $i$-finite. By definitions of $R_{i}(M)$ and $M$, the tuple $\left(y_{j}\right)_{j \in I}$ is a basis of $R_{i}(M)$, where

$$
y_{j}:= \begin{cases}y_{i} & \text { if } j=i, \\ \left(\operatorname{ad}_{c} x_{i}\right)^{-a_{i j}^{M}}\left(x_{j}\right) & \text { if } \quad j \neq i,\end{cases}
$$

where $y_{i} \in M_{i}^{*} \backslash\{0\}$.

From the method in [13, Example 1], one can obtain the labels of the Dynkin diagram of $R_{i}(M)=\left(R_{i}(M)_{j}\right)_{j \in I}$. In more detail, we have the following lemma.

Lemma 3.4. Let $i \in I$. Assume that $M$ is $i$-finite and let $a_{i j}:=a_{i j}^{M}$ for all $j \in I$. Let $\left(q_{j k}^{\prime}\right)_{j, k \in I}$ be the braiding matrix of $R_{i}(M)$ with respect to $\left(y_{j}\right)_{j \in I}$. Then the labels of the Dynkin diagram of $R_{i}(M)=\left(R_{i}(M)_{j}\right)_{j \in I}$ are

$$
\begin{aligned}
& q_{j j}^{\prime}= \begin{cases}q_{i i} & \text { if } j=i, \\
q_{j j} & \text { if } j \neq i, \quad q_{i j} q_{j i}=q_{i i}^{a_{i j}}, \\
q_{i i} q_{j j}\left(q_{i j} q_{j i}\right)^{-a_{i j}} & \text { if } j \neq i, \quad q_{i i} \in G_{1-a_{i j}}^{\prime}, \\
q_{j j}\left(q_{i j} q_{j i}\right)^{-a_{i j}} & \text { if } j \neq i, \quad q_{i i}=1,\end{cases} \\
& q_{i j}^{\prime} q_{j i}^{\prime}=\left\{\begin{array}{lll}
q_{i j} q_{j i} & \text { if } j \neq i, & q_{i j} q_{j i}=q_{i i}^{a_{i j}}, \\
q_{i i}^{2}\left(q_{i j} q_{j i}\right)^{-1} & \text { if } j \neq i, & q_{i i} \in G_{1-a_{i j}}^{\prime}, \\
\left(q_{i j} q_{j i}\right)^{-1} & \text { if } j \neq i, & q_{i i}=1,
\end{array}\right.
\end{aligned}
$$

and if $j, k \neq i, j \neq k$, then

$$
q_{j k}^{\prime} q_{k j}^{\prime}=\left\{\begin{array}{lll}
q_{j k} q_{k j} & \text { if } q_{i r} q_{r i}=q_{i i}^{a_{i r}}, \quad r \in\{j, k\}, \\
q_{j k} q_{k j}\left(q_{i k} q_{k i} q_{i i}^{-1}\right)^{-a_{i j}} & \text { if } q_{i j} q_{j i}=q_{i i}^{a_{i j}}, \quad q_{i i} \in G_{1-a_{i k}}^{\prime}, \\
q_{j k} q_{k j}\left(q_{i j} q_{j i}\right)^{-a_{i k}}\left(q_{i k} q_{k i}\right)^{-a_{i j}} & \text { if } q_{i i}=1, \\
q_{j k} q_{k j} q_{i i}^{2}\left(q_{i j} q_{j i} q_{i k} q_{k i}\right)^{-a_{i j}} & \text { if } q_{i i} \in G_{1-a_{i k}}^{\prime}, \quad q_{i i} \in G_{1-a_{i j}}^{\prime} .
\end{array}\right.
$$

Assume that $M$ admits all reflections. By Theorem 3.1, we are able to construct a semiCartan graph $\mathcal{C}(M)$ of $M$

$$
\mathcal{C}(M)=\left(I, \mathcal{X}_{\theta}^{\mathbb{k} G}(M),\left(r_{i}\right)_{i \in I},\left(A^{X}\right)_{X \in \mathcal{X}_{\theta}^{\mathbb{k} G}(M)}\right),
$$

where $\mathcal{X}_{\theta}^{\mathbb{k} G}(M)=\left\{\left[R_{i_{1}} \cdots R_{i_{n}}(M)\right] \in \mathcal{F}_{\theta}^{\mathbb{k} G} \mid n \in \mathbb{N}_{0}, i_{1}, \ldots, i_{n} \in I\right\}$. Note that any $X \in \mathcal{X}_{\theta}^{\mathbb{k} G}(M)$ has a well-defined braiding matrix given by the braiding matrix of any representative of $X$.

Definition 3.5. Assume that $M$ admits all reflections. For all $X \in \mathcal{X}_{\theta}^{\mathbb{k} G}(M)$ let

$$
[X]_{\theta}^{s}=\left\{Y \in \mathcal{X}_{\theta}^{\mathbb{k} G}(M) \mid Y \text { and } X \text { have the same Dynkin diagram }\right\} .
$$

Let $\mathcal{Y}_{\theta}^{s}(M)=\left\{[X]_{\theta}^{s} \mid X \in \mathcal{X}_{\theta}^{\mathbb{k} G}(M)\right\}$ and $A^{[X]_{\theta}^{s}}=A^{X}$ for all $X \in \mathcal{X}_{\theta}^{\mathbb{k} G}(M)$. Let $t: I \times \mathcal{Y}_{\theta}^{s}(M) \rightarrow$ $\mathcal{Y}_{\theta}^{s}(M),\left(i,[X]_{\theta}^{s}\right) \mapsto\left[r_{i}(X)\right]_{\theta}^{s}$. Then the tuple

$$
\mathcal{C}_{s}(M)=\left\{I, \mathcal{Y}_{\theta}^{s}(M), t,\left(A^{Y}\right)_{Y \in \mathcal{Y}_{\theta}^{s}(M)}\right\}
$$

is called the small semi-Cartan graph of $M$. 
Proposition 3.6. Assume that $M$ admits all reflections. Then the tuple

$$
\mathcal{C}_{s}(M)=\left\{I, \mathcal{Y}_{\theta}^{s}(M), t,\left(A^{Y}\right)_{Y \in \mathcal{Y}_{\theta}^{s}(M)}\right\}
$$

is a semi-Cartan graph. Moreover, if $\mathcal{C}(M)$ is a finite Cartan graph, then $\mathcal{C}_{s}(M)$ is a finite Cartan graph.

Proof. The map $t$ and $A^{[X]_{\theta}^{s}}$ are well-defined for all $[X]_{\theta}^{s} \in \mathcal{Y}_{\theta}^{s}(M)$. Indeed, if $X, X^{\prime} \in[X]_{\theta}^{s}$, then $A^{X}=A^{X^{\prime}}$ by Lemma 3.2. Thus $A^{[X]_{\theta}^{s}}$ is well-defined. Further, Lemma 3.4 implies that $r_{i}(X)$ and $r_{i}\left(X^{\prime}\right)$ have the same Dynkin diagram. Hence $t$ is well-defined. Since $t_{i}\left([X]_{\theta}^{s}\right)=$

$t\left(i,[X]_{\theta}^{s}\right)=\left[r_{i}(X)\right]_{\theta}^{s}$ and $r_{i}^{2}=\mathrm{id}$, then for all $i \in I, t_{i}^{2}=\operatorname{id}_{\mathcal{Y}_{\theta}^{s}(M)}$. Moreover, $a_{i j}^{[X]_{\theta}^{s}}=a_{i j}^{t_{i}\left([X]_{\theta}^{s}\right)}$. Hence the first claim holds.

The second claim follows from the definitions of $\boldsymbol{\Delta}^{X \text { re }}$ and $\mathcal{C}_{s}(M)$.

\section{Finite Cartan graphs of rank two}

In this section we simplify slightly the fundaments of the theory presented in [9] and give a characterization of finite Cartan graphs of rank two.

Definition 4.1. Let $\mathcal{A}^{+}$denote the smallest subset of $\cup_{n \geq 2} \mathbb{N}_{0}^{n}$ such that

- $(0,0) \in \mathcal{A}^{+}$,

- if $\left(c_{1}, \ldots, c_{n}\right) \in \mathcal{A}^{+}$and $1<i \leq n$, then $\left(c_{1}, \ldots, c_{i-2}, c_{i-1}+1,1, c_{i}+1, \ldots, c_{n}\right) \in \mathcal{A}^{+}$.

Remark 4.2. Note that our definition of $\mathcal{A}^{+}$is different from the one in [11].

From the definition of $\mathcal{A}^{+}$, we get the following lemma.

Lemma 4.3. Let $n \geq 2$ and $\left(c_{1}, \ldots, c_{n}\right) \in \mathcal{A}^{+}$. Then $\sum_{i=1}^{n} c_{i}=3 n-6$.

The definition of $\mathcal{A}^{+}$implies the following.

Proposition 4.4. Let $n \geq 2$. Enumerate the vertices of a convex $n$-gon by $1, \ldots, n$ such that consecutive integers correspond to neighboring vertices. Let $T_{n}$ be the set of triangulations of a convex $n$-gon with non-intersecting diagonals. Let $T=\cup_{n \geq 2} T_{n}$. For any triangulation $t \in T_{n}$ and any $i \in\{1, \ldots, n\}$, let $c_{i}$ be the number of triangles meeting at the $i$-th vertex. Then the map $\psi: T \rightarrow \mathcal{A}^{+}, t \mapsto\left(c_{1}, \ldots, c_{n}\right)$ is a bijection.

Proof. We proceed by induction on $n$. For $n=2$, a triangulation of a convex 2-gon is itself. Then $\left(c_{1}, c_{2}\right)=(0,0)$. Hence the claim is true for $n=2$. For $n \geq 3$, the definition of $\mathcal{A}^{+}$ corresponds bijectively to the construction of a triangulation of a convex $(n+1)$-gon by adding a new triangle between two consecutive vertices of a convex $n$-gon, but not at the edge between the first and the last vertex. By adding one triangle between two consecutive vertices of a convex $n$-gon, one increases the number of triangles at the two adjacent vertices and the number of triangles at the new vertex becomes 1 .

Corollary 4.5. Let $n \geq 2$ and let $\left(c_{1}, \ldots, c_{n}\right) \in \mathcal{A}^{+}$.

(1) $\left(c_{n}, c_{n-1}, \ldots, c_{1}\right) \in \mathcal{A}^{+}$and $\left(c_{2}, c_{3}, \ldots, c_{n}, c_{1}\right) \in \mathcal{A}^{+}$.

(2) If $n \geq 3$, then there is $1<i<n$ satisfying $c_{i}=1$. For any such $i,\left(c_{1}, \ldots, c_{i-2}, c_{i-1}-1\right.$, $\left.c_{i+1}-1, c_{i+2}, \ldots, c_{n}\right) \in \mathcal{A}^{+}$.

(3) If $n \geq 3$, then $c_{i} \geq 1$ for all $1 \leq i \leq n$.

(4) If $c_{i}=1, c_{i+1}=1$ for some $1 \leq i \leq n-1$, then $n=3$ and $c=(1,1,1)$. 
Table 4.1. Sequences in $\mathcal{A}^{+}$containing exactly one subsequence from Theorem 4.6.

\begin{tabular}{r|r}
\hline \multicolumn{1}{|c|}{ subsequences } & sequences in $\mathcal{A}^{+}$ \\
\hline \hline$(1,1)$ & $(1,1,1)$ \\
\hline$(1,2,1)$ & $(1,2,1,2)$ \\
\hline$(1,2,2)$ & $(1,2,2,2,2,2,1,6)$ \\
\hline$(1,2,3)$ & $(1,2,3,1,6,1,2,3,1,6,1,2,3,1,6)$ \\
\hline$(2,1,3)$ & $(2,1,3,4,2,1,3,4,2,1,3,4)$ \\
\hline$(2,1,4)$ & $(2,1,4,2,1,4,2,1,4)$ \\
\hline$(2,1,5)$ & $(2,1,5,1,2,4,2,1,5,1,2,4)$ \\
\hline$(1,3,1,3)$ & $(1,3,1,3,1,3)$ \\
\hline$(1,3,1,4)$ & $(1,3,1,4,1,3,1,4)$ \\
\hline$(1,3,1,5)$ & $(1,3,1,5,1,3,1,5,1,3,1,5)$ \\
\hline
\end{tabular}

Proof. (1) and (2) follow directly from the bijection between $\mathcal{A}^{+}$and triangulations of convex $n$ gons in Proposition 4.4. (3) follows from the definition of $\mathcal{A}^{+}$. (4) follows from (2) and (3).

We say that two consecutive entries of a sequence in $\mathcal{A}^{+}$are neighbors.

Theorem 4.6. Let $n \geq 3$. Then any sequence $\left(c_{1}, \ldots, c_{n}\right) \in \mathcal{A}^{+}$contains a subsequence $\left(c_{k}\right)_{i \leq k \leq j}$, where $1 \leq i \leq j \leq n$, of the form

$$
(1,1), \quad(1,2, a), \quad(2,1, b), \quad(1,3,1, b)
$$

or their transpose, where $1 \leq a \leq 3$ and $3 \leq b \leq 5$.

Remark 4.7. We record that it is natural to exclude the cases $b=1$ and $b=2$ since $(1,3,1,1)$ contains the subsequence $(1,1)$ and $(1,3,1,2)$ contains the transpose of $(2,1,3)$.

Remark 4.8. The claim becomes false by omitting one of the sequences from the theorem. In Table 4.1 we list sequences in $\mathcal{A}^{+}$which contain precisely one of the sequences in Theorem 4.6.

Proof. Let $c=\left(c_{1}, \ldots, c_{n}\right) \in \mathcal{A}^{+}$such that the claim does not hold for $c$. Then $n \geq 5$ and $c$ has no subsequence $(2,1,2)$. Otherwise $c=(1,2,1,2)$ or $c=(2,1,2,1)$ by Corollary $4.5(2),(4)$. We define $E=\left\{\nu_{i j} \mid i, j \in\{1,2\}\right\}$, where the sequences $\nu_{i j}$ are given by

$$
\nu_{11}=(1), \quad \nu_{12}=(2,1), \quad \nu_{21}=(1,2), \quad \nu_{22}=(1,3,1) .
$$

Now we decompose $c$ by the following steps.

Replace all subsequences $(2,1)$ by $\nu_{12}$, then all subsequences $(1,2)$ by $\nu_{21}$, then all subsequences $(1,3,1)$ by $\nu_{22}$, and finally all entries 1 by $\nu_{11}$. By this construction, $\left(\nu_{11}, 3, \nu_{11}\right)$ is not a subsequence of $d$. Hence we get a decomposition $d=\left(d_{1}, \ldots, d_{k}\right)$, where $k \geq 2$, of $c$ into subsequences of the form $(a)$ and $\nu$, where $a \geq 2$ and $\nu \in E$.

Since $(1,1),(1,2, a),(2,1, b),(1,3,1, b)$ and their transposes are not subsequences of $c$, where $1 \leq a \leq 3,2 \leq b \leq 5$, we obtain the following conditions on the entries of $d$ :

- no entry $\nu_{i j}$ of $d$, where $i, j \in\{1,2\}$, has 2 or $\nu_{k l}$ with $k, l \in\{1,2\}$ as a neighbor.

- if $\left(\nu_{21}, a\right)$ or $\left(a, \nu_{12}\right)$ is a subsequence of $d$, then $a \geq 4$.

- if $\left(\nu_{i 2}, b\right)$ or $\left(b, \nu_{2 i}\right)$ is a subsequence of $d$, where $i \in\{1,2\}$, then $b \geq 6$.

By applying Corollary 4.5(2) we get further reductions of $d$ :

$$
\left(\ldots, d_{m-1}, \nu_{i j}, d_{m+1}, \ldots\right) \rightarrow\left(\ldots, d_{m-1}-i, d_{m+1}-j\right), \quad\left(\nu_{i 2}, d_{2}, \ldots\right) \rightarrow\left(\nu_{i 1}, d_{2}-1, \ldots\right),
$$


where $i, j \in\{1,2\}$. Thus we can perform such reductions at all places in $d$, where an entry $\nu_{i j}$ with $i, j \in\{1,2\}$ appears. After decreasing them, we get $d_{m} \geq 2$, where $1<m<k$. Indeed, we get the following conditions.

- If $d=\left(\ldots, d_{m-1}, d_{m}, d_{m+1}, \ldots\right)$, where $d_{m} \geq 6$, then $d_{m}$ can be reduced at most by 4 . Hence the value of $d_{m}$ after reduction is at least 2 .

- If $4 \leq d_{m} \leq 5$, then neither $\left(\nu_{i 2}, d_{m}\right)$ nor $\left(d_{m}, \nu_{2 i}\right)$ is a subsequence of $d$, where $i \in\{1,2\}$. Hence $d$ can be reduced by at most 2 .

- If $d_{m}=3$, then $d_{m-1}, d_{m+1} \notin\left\{\nu_{12}, \nu_{21}, \nu_{22}\right\}$. Further, $\left(d_{m-1}, d_{m+1}\right) \neq\left(\nu_{11}, \nu_{11}\right)$. Hence $d_{m}$ decreases by at most 1 .

- If $d_{m}=2$, then it has no neighbour $\nu_{i j}$ with $i, j \in\{1,2\}$. Hence $d_{m}$ does not change.

Thus one can reduce $c$ to a sequence $\left(c_{1}^{\prime}, \ldots, c_{l}^{\prime}\right)$ with $l \geq 1$, where $c_{m}^{\prime} \geq 2$ for all $1<m<l$ and $c_{1}^{\prime}, c_{l}^{\prime} \geq 1$. This is a contradiction to Corollary $4.5(2)$.

Recall that $\left(\alpha_{1}, \alpha_{2}\right)$ is the standard basis of $\mathbb{Z}^{2}$. We define a map

$$
\eta: \mathbb{Z} \rightarrow \operatorname{SL}(2, \mathbb{Z}), \quad a \mapsto\left(\begin{array}{cc}
a & -1 \\
1 & 0
\end{array}\right) .
$$

Lemma 4.9. Let $n \in \mathbb{N}$ and $\left(c_{k}\right)_{1 \leq k \leq n} \in \mathbb{Z}^{n}$. For all $1 \leq k \leq n+1$, let $\beta_{0}=-\alpha_{2}$ and $\beta_{k}=\eta\left(c_{1}\right) \ldots \eta\left(c_{k-1}\right)\left(\alpha_{1}\right)$. Then the following hold:

(1) $\beta_{k+1}=c_{k} \beta_{k}-\beta_{k-1}$ for all $1 \leq k \leq n$,

(2) if $c_{1} \geq 1$ and $c_{k} \geq 2$ for all $1<k<n$, then $\beta_{k} \in \mathbb{N}_{0}^{2}$ for all $1 \leq k \leq n$ and $\beta_{k}-\beta_{k-1} \in$ $\mathbb{N}_{0}^{2} \backslash\{0\}$ for $1<k \leq n$.

Proof. (1) By definition, $\beta_{1}=\alpha_{1}$ and $\beta_{2}=\eta\left(c_{1}\right)\left(\alpha_{1}\right)=c_{1} \alpha_{1}+\alpha_{2}$. Thus the claim holds for $k=1$. Since $\eta\left(c_{k-1}\right)\left(\alpha_{2}\right)=-\alpha_{1}$, then

$$
\beta_{k+1}=\eta\left(c_{1}\right) \ldots \eta\left(c_{k}\right)\left(\alpha_{1}\right)=\eta\left(c_{1}\right) \ldots \eta\left(c_{k-1}\right)\left(c_{k} \alpha_{1}+\alpha_{2}\right)=c_{k} \beta_{k}-\beta_{k-1}
$$

for all $k \geq 2$.

(2) For all $0 \leq k \leq n$, let $a_{k}, b_{k} \in \mathbb{Z}$ such that $\beta_{k}=a_{k} \alpha_{1}+b_{k} \alpha_{2}$. By induction on $k$, we get the following.

- If $c_{k} \geq 2$ for $1 \leq k<n$, then

$$
a_{k}>b_{k} \geq 0, \quad a_{k}>a_{k-1}, \quad b_{k}>b_{k-1}, \quad a_{k}-b_{k}-\left(a_{k-1}-b_{k-1}\right) \geq 0
$$

for all $1 \leq k \leq n$.

- If $c_{1}=1$ and $c_{k} \geq 2$ for $2 \leq k<n$, then

$$
b_{k} \geq a_{k}>0, \quad a_{k} \geq a_{k-1}, \quad b_{k}>b_{k-1}, \quad a_{k}-b_{k}-\left(a_{k-1}-b_{k-1}\right)<0 .
$$

for all $2 \leq k \leq n$.

Thus $\beta_{k} \in \mathbb{N}_{0}^{2}$ for all $1 \leq k \leq n$ and $\beta_{k}-\beta_{k-1} \in \mathbb{N}_{0}^{2} \backslash\{0\}$ for $1<k \leq n$.

The following theorem will be used in the proof of Theorem 4.15. It was proven partially in $[9$, Propositon 5.3]. Notice that the definition of $\mathcal{A}^{+}$is different from the one in [9].

Theorem 4.10. Let $n \geq 2$ and $\left(c_{i}\right)_{1<i<n} \in \mathbb{Z}^{n}$. Then the following are equivalent:

(1) $\left(c_{i}\right)_{1 \leq i \leq n} \in \mathcal{A}^{+}$,

(2) $\eta\left(c_{1}\right) \cdots \eta\left(c_{n}\right)=-$ id and $\beta_{k}=\eta\left(c_{1}\right) \cdots \eta\left(c_{k-1}\right)\left(\alpha_{1}\right) \in \mathbb{N}_{0}^{2}$ for all $1 \leq k \leq n$. 
Proof. $(1) \Rightarrow(2)$. We apply induction on $n$. If $n=2$, then $\left(c_{1}, c_{2}\right)=(0,0), \eta(0)^{2}=-\mathrm{id}, \beta_{1}=\alpha_{1}$, and $\beta_{2}=\alpha_{2}$. Assume that $n \geq 3$. By the definition of $\mathcal{A}^{+}$, there is a $\left(c_{1}^{\prime}, \ldots, c_{n-1}^{\prime}\right) \in \mathcal{A}^{+}$and $1<i \leq n-1$ such that

$$
\left(c_{1}, \ldots, c_{n}\right)=\left(c_{1}^{\prime}, \ldots, c_{i-1}^{\prime}+1,1, c_{i}^{\prime}+1, c_{i+1}^{\prime}, \ldots, c_{n-1}^{\prime}\right) .
$$

By calculation,

$$
\eta(a) \eta(b)=\eta(a+1) \eta(1) \eta(b+1)
$$

for all $a, b \in \mathbb{Z}$. Then

$$
\eta\left(c_{1}\right) \cdots \eta\left(c_{n}\right)=\eta\left(c_{1}^{\prime}\right) \cdots \eta\left(c_{n-1}^{\prime}\right)=-\mathrm{id} .
$$

Let $\beta_{i}^{\prime}=\eta\left(c_{1}^{\prime}\right) \cdots \eta\left(c_{i-1}^{\prime}\right)\left(\alpha_{1}\right)$ for all $1 \leq i \leq n-1$. Then $\beta_{k}=\beta_{k}^{\prime}$ for all $1 \leq k<i$ and $\beta_{k}=\beta_{k-1}^{\prime}$ for all $i+1 \leq k \leq n+1$. Finally

$$
\begin{aligned}
\beta_{i} & =\eta\left(c_{1}\right) \cdots \eta\left(c_{i-1}\right)\left(\alpha_{1}\right)=\eta\left(c_{1}^{\prime}\right) \cdots \eta\left(c_{i-2}^{\prime}\right) \eta\left(c_{i-1}^{\prime}+1\right)\left(\alpha_{1}\right) \\
& =\eta\left(c_{1}^{\prime}\right) \cdots \eta\left(c_{i-2}^{\prime}\right)\left(\eta\left(c_{i-1}^{\prime}\right)\left(\alpha_{1}\right)+\alpha_{1}\right)=\beta_{i}^{\prime}+\beta_{i-1}^{\prime} \in \mathbb{N}_{0}^{2} .
\end{aligned}
$$

Then (2) follows.

$(2) \Rightarrow(1)$. Again we proceed by induction. If $n=2$, then

$$
\eta\left(c_{1}\right) \eta\left(c_{2}\right)=\left(\begin{array}{cc}
c_{1} c_{2}-1 & -c_{1} \\
c_{2} & -1
\end{array}\right)=-\mathrm{id}
$$

implies that $\left(c_{1}, c_{2}\right)=(0,0) \in \mathcal{A}^{+}$. Assume that $n \geq 3$. Set $\beta_{0}=-\alpha_{2}$. One has $\beta_{k+1}=$ $c_{k} \beta_{k}-\beta_{k-1}$ for all $1 \leq k<n$. By assumption, the condition $\beta_{k-1}, \beta_{k}, \beta_{k+1} \in \mathbb{N}_{0}^{2}$ implies $c_{k}>0$ for $2 \leq k<n$ and $c_{1} \geq 0$. If $c_{1}=0$ then $\beta_{2}=\alpha_{2}$ and $\beta_{3}=c_{2} \alpha_{2}-\alpha_{1} \notin \mathbb{N}_{0}^{2}$. Hence $c_{k} \geq 1$ for all $1 \leq k<n$. Moreover, there is $1<i<n$ satisfying $c_{i}=1$. Indeed, $\beta_{n+1}=c_{n} \beta_{n}-\beta_{n-1}=\left(c_{n}-1\right) \beta_{n}+\left(\beta_{n}-\beta_{n-1}\right)$ by Lemma 4.9(1). Assume that $c_{i} \geq 2$ for all $1<i<n$. Then $\beta_{n+1} \in \mathbb{N}_{0}^{2}$ if $c_{n} \geq 1$ and $-\beta_{n+1} \in \mathbb{N}_{0}^{2} \backslash\left\{0, \alpha_{1}\right\}$ if $c_{n} \leq 0$ by Lemma $4.9(2)$, since $n \geq 3$. This is a contradiction to $\beta_{n+1}=\eta\left(c_{1}\right) \cdots \eta\left(c_{n}\right)\left(\alpha_{1}\right)=(-\mathrm{id})\left(\alpha_{1}\right)=-\alpha_{1}$.

Hence there is $\left(c_{1}^{\prime}, \ldots, c_{n-1}^{\prime}\right) \in \mathbb{Z}^{n-1}$ such that

$$
\left(c_{1}, \ldots, c_{n}\right)=\left(c_{1}^{\prime}, \ldots, c_{i-1}^{\prime}+1,1, c_{i}^{\prime}+1, c_{i+1}^{\prime}, \ldots, c_{n-1}^{\prime}\right) .
$$

Then $\eta\left(c_{1}\right) \cdots \eta\left(c_{n}\right)=\eta\left(c_{1}^{\prime}\right) \cdots \eta\left(c_{n-1}^{\prime}\right)=-$ id by equation $(4.2)$ and $\beta_{k}^{\prime}=\eta\left(c_{1}^{\prime}\right) \ldots \eta\left(c_{k-1}^{\prime}\right)\left(\alpha_{1}\right) \in$ $\mathbb{N}_{0}^{2}$ for all $1 \leq k \leq n-1$. Hence $\left(c_{1}^{\prime}, \ldots, c_{n-1}^{\prime}\right) \in \mathcal{A}^{+}$by induction hypothesis. Then

$$
\left(c_{1}, \ldots, c_{n}\right) \in \mathcal{A}^{+} \text {. }
$$

Definition 4.11. Let $\mathcal{C}=\mathcal{C}\left(I, \mathcal{X}, r,\left(A^{X}\right)_{X \in \mathcal{X}}\right)$ be a semi-Cartan graph of rank two and let $X \in \mathcal{X}$ and $i \in I$. The characteristic sequence of $\mathcal{C}$ with respect to $X$ and $i$ is the infinite sequence $\left(c_{k}^{X, i}\right)_{k \geq 1}$ of non-negative integers, where

$$
c_{2 k+1}^{X, i}=-a_{i j}^{\left(r_{j} r_{i}\right)^{k}(X)}=-a_{i j}^{r_{i}\left(r_{j} r_{i}\right)^{k}(X)}, \quad c_{2 k+2}^{X, i}=-a_{j i}^{r_{i}\left(r_{j} r_{i}\right)^{k}(X)}=-a_{j i}^{\left(r_{j} r_{i}\right)^{k+1}(X)}
$$

for all $k \geq 0$ and $j \in I \backslash\{i\}$.

By the definition of a characteristic sequence, we get the following remark.

Remark 4.12. Let $\mathcal{C}=\mathcal{C}\left(I, \mathcal{X}, r,\left(A^{X}\right)_{X \in \mathcal{X}}\right)$ be a semi-Cartan graph of rank two and let $X \in \mathcal{X}$ and $i, j \in I$ with $i \neq j$. Let $\left(c_{k}\right)_{k \geq 1}$ be the characteristic sequence of $\mathcal{C}$ with respect to $X$ and $i$. 
- The characteristic sequence of $\mathcal{C}$ with respect to $r_{i}(X)$ and $j$ is $\left(c_{k+1}\right)_{k \geq 1}$.

- Suppose that $\left(r_{j} r_{i}\right)^{n}(X)=X$ for some $n \geq 1$. Then the characteristic sequence of $\mathcal{C}$ with respect to $X$ and $j$ is $\left(c_{2 n+1-k}\right)_{k \geq 1}$.

Definition 4.13. Let $\mathcal{C}=\mathcal{C}\left(I, \mathcal{X}, r,\left(A^{X}\right)_{X \in \mathcal{X}}\right)$ be a semi-Cartan graph of rank two and let $X \in \mathcal{X}$ and $i \in I$. Let $\left(c_{k}\right)_{k \geq 1}$ be the characteristic sequence of $\mathcal{C}$ with respect to $X$ and $i$. The root sequence of $\mathcal{C}$ with respect to $X$ and $i$ is the infinite sequence $\left(\beta_{k}\right)_{k \geq 1}$ of elements of $\mathbb{Z}^{2}$, where

$$
\beta_{k}=\eta\left(c_{1}\right) \cdots \eta\left(c_{k-1}\right)\left(\alpha_{1}\right)
$$

for all $k \geq 1$. In particular, $\beta_{1}=\alpha_{1}$.

Let $\mathcal{C}=\mathcal{C}\left(I=\{1,2\}, \mathcal{X}, r,\left(A^{X}\right)_{X \in \mathcal{X}}\right)$ be a semi-Cartan graph. For all $X \in \mathcal{X}$, the maps $s_{1}^{X}, s_{2}^{X}$ are defined by equation (2.1). Recall that $\left(\alpha_{1}, \alpha_{2}\right)$ is a basis of $\mathbb{Z}^{2}$ and $\eta$ is a map defined by equation (4.1). Define a map

$$
\tau: \mathbb{Z}^{2} \rightarrow \mathbb{Z}^{2}, \quad a \alpha_{1}+b \alpha_{2} \mapsto b \alpha_{1}+a \alpha_{2}
$$

for any $a, b \in \mathbb{Z}$. One obtains

$$
s_{1}^{X}=\eta\left(-a_{12}^{X}\right) \tau, \quad s_{2}^{X}=\tau \eta\left(-a_{21}^{X}\right)
$$

for all $X \in \mathcal{X}$.

Lemma 4.14. Let $\mathcal{C}=\mathcal{C}\left(I=\{1,2\}, \mathcal{X}, r,\left(A^{X}\right)_{X \in \mathcal{X}}\right)$ be a semi-Cartan graph of rank two and let $X \in \mathcal{X}$. Let $\left(\beta_{k}\right)_{k \geq 1}$ be the root sequence of $\mathcal{C}$ with respect to $X$ and 1 and let $\left(\gamma_{k}\right)_{k \geq 1}$ be the root sequence of $\mathcal{C}$ with respect to $X$ and 2 . Then

$$
\begin{array}{ll}
\beta_{2 k+1}=\operatorname{id}_{X}\left(s_{1} s_{2}\right)^{k} \alpha_{1}, & \beta_{2 k+2}=\operatorname{id}_{X}\left(s_{1} s_{2}\right)^{k} s_{1} \alpha_{2}, \\
\tau \gamma_{2 k+1}=\operatorname{id}_{X}\left(s_{2} s_{1}\right)^{k} \alpha_{2}, & \tau \gamma_{2 k+2}=\operatorname{id}_{X}\left(s_{2} s_{1}\right)^{k} s_{2} \alpha_{1}
\end{array}
$$

for all $k \geq 0$. Hence $\boldsymbol{\Delta}^{X \mathrm{re}}=\left\{ \pm \beta_{k}, \pm \tau \gamma_{k} \mid k \geq 1\right\}$.

Proof. Let $\left(c_{k}\right)_{k \geq 1}$ be the characteristic sequence with respect to $X$ and $i=1$. By equation (4.3) and the definition of the root sequence, one obtains that

$$
\begin{aligned}
\beta_{2 k+1} & =\eta\left(c_{1}\right) \eta\left(c_{2}\right) \cdots \eta\left(c_{2 k-1}\right) \eta\left(c_{2 k}\right)\left(\alpha_{1}\right) \\
& =\eta\left(-a_{12}^{X}\right) \eta\left(-a_{21}^{r_{1}(X)}\right) \cdots \eta\left(-a_{12}^{\left(r_{2} r_{1}\right)^{k-1}(X)}\right) \eta\left(-a_{21}^{r_{1}\left(r_{2} r_{1}\right)^{k-1}(X)}\right)\left(\alpha_{1}\right) \\
& =\eta\left(-a_{12}^{X}\right) \tau \tau \eta\left(-a_{21}^{r_{1}(X)}\right) \cdots \eta\left(-a_{12}^{\left(r_{2} r_{1}\right)^{k-1}(X)}\right) \tau \tau \eta\left(-a_{21}^{r_{1}\left(r_{2} r_{1}\right)^{k-1}(X)}\right)\left(\alpha_{1}\right) \\
& =s_{1}^{X} s_{2}^{r_{1}(X)} \cdots s_{1}^{\left(r_{2} r_{1}\right)^{k-1}(X)} s_{2}^{r_{1}\left(r_{2} r_{1}\right)^{k-1}(X)}\left(\alpha_{1}\right)=\operatorname{id}_{X}\left(s_{1} s_{2}\right)^{k} \alpha_{1}, \\
\tau \gamma_{2 k+1} & =\tau \eta\left(-a_{21}^{X}\right) \eta\left(-a_{12}^{r_{2}(X)}\right) \cdots \eta\left(-a_{21}^{\left(r_{1} r_{2}\right)^{n-2}(X)}\right) \eta\left(-a_{12}^{r_{2}\left(r_{1} r_{2}\right)^{k-1}(X)}\right)\left(\alpha_{1}\right) \\
& =\left(\tau \eta\left(-a_{21}^{X}\right) \eta\left(-a_{12}^{r_{2}(X)}\right) \tau\right)(\tau \cdots \tau)\left(\tau \eta\left(-a_{21}^{\left(r_{1} r_{2}\right)^{k-2}(X)}\right) \eta\left(-a_{12}^{r_{2}\left(r_{1} r_{2}\right)^{k-1}(X)}\right) \tau\right) \tau\left(\alpha_{1}\right) \\
& =\operatorname{id}_{X}\left(s_{2} s_{1}\right)^{k} \alpha_{2} .
\end{aligned}
$$

The claims $\beta_{2 k+2}=\operatorname{id}_{X}\left(s_{1} s_{2}\right)^{k} s_{1} \alpha_{2}, \tau \gamma_{2 k+2}=\operatorname{id}_{X}\left(s_{2} s_{1}\right)^{k} s_{2} \alpha_{1}$ hold by a similar argument. Thus $\boldsymbol{\Delta}^{X \mathrm{re}}=\left\{ \pm \beta_{k}, \pm \tau \gamma_{k} \mid k \geq 1\right\}$ follows from the definition of $\boldsymbol{\Delta}^{X \mathrm{re}}$.

For a finite sequence $\left(v_{1}, \ldots, v_{n}\right)$ of integers or vectors, where $n \geq 1$, let $\left(v_{1}, \ldots, v_{n}\right)^{\infty}=$ $\left(u_{k}\right)_{k \geq 1}$ be the sequence where $u_{m n+i}=v_{i}$ for all $1 \leq i \leq n, m \geq 0$. 
Theorem 4.15. Let $\mathcal{C}=\mathcal{C}\left(I=\{1,2\}, \mathcal{X}, r,\left(A^{X}\right)_{X \in \mathcal{X}}\right)$ be a connected semi-Cartan graph of rank two such that $|\mathcal{X}|$ is finite. Let $X \in \mathcal{X}$ and let $n$ be the smallest positive integer with $\left(r_{2} r_{1}\right)^{n}(X)=X$. Let $\left(c_{k}\right)_{k \geq 1}$ be the characteristic sequence of $\mathcal{C}$ with respect to $X$ and 1 , and let $l=6 n-\sum_{i=1}^{2 n} c_{i}$. The following are equivalent:

(1) $\mathcal{C}$ is a finite Cartan graph,

(2) $l>0, l \mid 12,\left(c_{1}, c_{2}, \ldots, c_{12 n / l}\right) \in \mathcal{A}^{+}$, and $\left(c_{k}\right)_{k \geq 1}=\left(c_{1}, c_{2}, \ldots, c_{12 n / l}\right)^{\infty}$.

In this case $12 n / l=\left|\boldsymbol{\Delta}_{+}^{X \mathrm{re}}\right|=t_{12}^{X}$.

Proof. Let $\left(\beta_{k}\right)_{k \geq 1}$ be the root sequence of $\mathcal{C}$ with respect to $X$ and 1 and $\left(\gamma_{k}\right)_{k \geq 1}$ the root sequence of $\mathcal{C}$ with respect to $X$ and 2 .

$(1) \Rightarrow(2)$. Let $q=t_{12}^{X}$. Then $\boldsymbol{\Delta}^{Y \text { re }} \subset \mathbb{N}_{0}^{2} \cup-\mathbb{N}_{0}^{2}$ for all $Y \in \mathcal{X}$ since $\mathcal{C}$ is a Cartan graph. By [16, Lemmas 3,4] and Lemma 4.14, we have $\beta_{k} \in \mathbb{N}_{0}^{2}$ for all $1 \leq k \leq q$ and $\beta_{q}=\eta\left(c_{1}\right) \cdots \eta\left(c_{q-1}\right)\left(\alpha_{1}\right)=\alpha_{2}$. By the same reason we obtain that $\eta\left(c_{2}\right) \cdots \eta\left(c_{q}\right)\left(\alpha_{1}\right)=\alpha_{2}$. Then we have

$$
-\beta_{q+1}=-\eta\left(c_{1}\right) \cdots \eta\left(c_{q}\right)\left(\alpha_{1}\right)=-\eta\left(c_{1}\right)\left(\alpha_{2}\right)=\alpha_{1} .
$$

Thus $-\eta\left(c_{1}\right) \cdots \eta\left(c_{q}\right)=\mathrm{id}$. Indeed, if we set $w:=-\eta\left(c_{1}\right) \ldots \eta\left(c_{q}\right)$ and $w\left(\alpha_{2}\right):=a \alpha_{1}+b \alpha_{2}$, then $b=1$ since $\operatorname{det}(w)=1$. If $q$ is odd then $-w \tau \in \operatorname{Hom}(Y, X)$ by equation (4.1) and equation (4.3), where $Y=r_{1}\left(r_{2} r_{1}\right)^{(q-1) / 2}(X)$. In the same way, one gets $-w \in \operatorname{Hom}\left(\left(r_{2} r_{1}\right)^{q / 2}(X), X\right)$ if $q$ is even. Hence $w\left(\alpha_{1}\right), w\left(\alpha_{2}\right) \in \boldsymbol{\Delta}^{X \mathrm{re}}$. Then $a \geq 0$ since $w\left(\alpha_{2}\right)=\eta\left(c_{1}\right) \cdots \eta\left(c_{q-1}\right)\left(\alpha_{1}\right)=\beta_{q} \in$ $\boldsymbol{\Delta}^{X \mathrm{re}} \subset \mathbb{N}_{0}^{2} \cup-\mathbb{N}_{0}^{2}$. Moreover, $a \leq 0$ since $w^{-1}\left(\alpha_{2}\right)=\alpha_{2}-a \alpha_{1} \in \mathbb{N}_{0}^{2} \cup-\mathbb{N}_{0}^{2}$. Hence $a=0$ and $w\left(\alpha_{2}\right)=\alpha_{2}$. Then $-\eta\left(c_{1}\right) \cdots \eta\left(c_{q}\right)=\mathrm{id}$. Hence $\left(c_{1}, \ldots, c_{q}\right) \in \mathcal{A}^{+}$by Theorem 4.10. Therefore $\sum_{i=1}^{q} c_{i}=3 q-6$ by Lemma 4.3 .

Further, we apply the first part of the proof for $r_{1}(X)$ and the label 2 instead of $X$ and the label 1 , respectively. Then $\left(c_{2}, \ldots, c_{q+1}\right) \in \mathcal{A}^{+}$by Remark 4.12 and $\sum_{i=2}^{q+1} c_{i}=3 q-6$. Hence $c_{q+1}=c_{1}$. By induction, $\left(c_{k}\right)_{k \geq 1}=\left(c_{1}, c_{2}, \ldots, c_{q}\right)^{\infty}$. In particular, we obtain that $\sum_{i=1}^{2 q n} c_{i}=$ $2 n(3 q-6)=q\left(\sum_{i=1}^{2 n} c_{i}\right)$. Therefore $\sum_{i=1}^{2 n} c_{i}=6 n-12 n / q$. Hence $q \mid 12 n$ and $l=6 n-\sum_{i=1}^{2 n} c_{i}=$ $12 n / q>0$. Further, $n \mid q$ since $\mathcal{C}$ is a Cartan graph. Thus $l \mid 12$.

$(2) \Rightarrow(1)$. Set $q=12 n / l$. Then $\eta\left(c_{1}\right) \ldots \eta\left(c_{q}\right)=-$ id and $\beta_{k} \in \mathbb{N}_{0}^{2}$ for $1 \leq k \leq q$ by Theorem 4.10. Then $\left(c_{q}, c_{q-1}, \ldots, c_{1}\right) \in \mathcal{A}^{+}$by Corollary $4.5(1)$ since $\left(c_{1}, c_{2}, \ldots, c_{q}\right) \in \mathcal{A}^{+}$. Since $l \mid 12, q$ is a multiple of $n$. Hence $\left(c_{q}, c_{q-1}, \ldots, c_{1}\right)^{\infty}$ is the characteristic sequence of $\mathcal{C}$ with respect to $X$ and 2. By Lemma 4.14, we get that $\gamma_{k} \in \mathbb{N}_{0}^{2}$ for all $1 \leq k \leq q$. Therefore, since $\left(c_{k}\right)_{k \geq 1}=\left(c_{1}, \ldots, c_{q}\right)^{\infty}, \Delta^{X \mathrm{re}}=\left\{ \pm \beta_{k}, \pm \tau \gamma_{k} \mid 1 \leq k \leq q\right\} \subseteq \mathbb{N}_{0}^{2} \cup-\mathbb{N}_{0}^{2}$ for all $X \in \mathcal{X}$ by Lemma 4.14. Hence $\mathcal{C}$ is finite.

By the definition of $t_{12}^{X}$ and [16, Lemma 4], we obtain that $t_{12}^{X}=q=\left|\Delta_{+}^{X \text { ree }}\right|$. Hence $n \mid t_{12}^{X}$ by assumption and $\left(r_{2} r_{1}\right)^{t_{12}^{X}}(X)=X$. Therefore, $\mathcal{C}$ is a Cartan graph.

\section{Classification of rank two Nichols algebras of diagonal type over fields of positive characteristic}

In this section, we classify all the two-dimensional braided vector spaces $V$ of diagonal type over fields of positive characteristic such that the Nichols algebra of $V$ has a finite root system. The proof uses the characterization of the finite Cartan graphs of rank two. 
Table 5.1. Dynkin diagrams in characteristic $p=2$.

\begin{tabular}{|c|c|c|}
\hline & Dynkin diagrams & fixed parameters \\
\hline 1 & $\begin{array}{rr}q & r \\
\bigcirc & \bigcirc \\
\end{array}$ & $q, r \in \mathbb{k}^{*}$ \\
\hline 2 & $\stackrel{q}{q} q^{-1} \quad{ }^{q}$ & $q \in \mathbb{K}^{*} \backslash\{1\}$ \\
\hline 3 & $\begin{array}{cccccc}q & q^{-1} & 1 & 1 & q & 1 \\
\bigcirc & & \bigcirc & \bigcirc & & \\
& \bigcirc & & & \end{array}$ & $q \in \mathbb{k}^{*} \backslash\{1\}$ \\
\hline 4 & $\begin{array}{lll}q & q^{-2} & q^{2} \\
\bigcirc & \bigcirc\end{array}$ & $q \in \mathbb{k}^{*} \backslash\{1\}$ \\
\hline 5 & $\begin{array}{cccccc}q & q^{-2} & 1 & q^{-1} & q^{2} & 1 \\
\bigcirc & & \bigcirc & \bigcirc & & \\
\end{array}$ & $q \in \mathbb{k}^{*} \backslash\{1\}$ \\
\hline 6 & $\bigcirc^{\zeta} q^{-1} \quad q \quad \zeta \quad \zeta^{-1} q \zeta q^{-1}$ & $\zeta \in G_{3}^{\prime}, q \in \mathbb{R}^{*} \backslash\left\{1, \zeta, \zeta^{2}\right\}$ \\
\hline 7 & 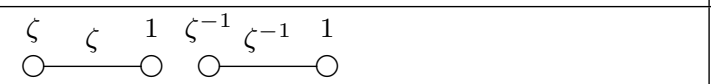 & $\zeta \in G_{3}^{\prime}$ \\
\hline 10 & 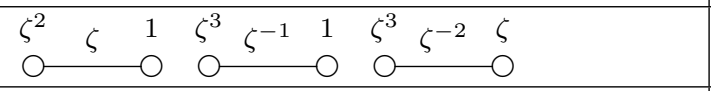 & $\zeta \in G_{9}^{\prime}$ \\
\hline 11 & $\begin{array}{lll}q & q^{-3} & q^{3} \\
\bigcirc\end{array}$ & $q \in \mathbb{k}^{*} \backslash\{1\}, q \notin G_{3}^{\prime}$ \\
\hline 14 & 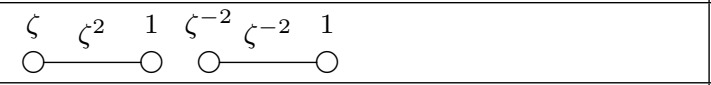 & $\zeta \in G_{5}^{\prime}$ \\
\hline 16 & \begin{tabular}{cccccccccccc}
$\zeta^{5}$ & $\zeta^{-3}$ & $\zeta$ & $\zeta^{5}$ & $\zeta^{-2}$ & 1 & $\zeta^{3}$ & $\zeta^{2}$ & 1 & $\zeta^{3}$ & $\zeta^{4}$ & $\zeta^{-4}$ \\
\hdashline & & $\bigcirc$ & $\bigcirc$ & & $\bigcirc$ & $\bigcirc$ & & $\bigcirc$ & $\bigcirc$ & & $\bigcirc$ \\
\end{tabular} & $\zeta \in G_{15}^{\prime}$ \\
\hline 17 & \begin{tabular}{cccccc}
$\zeta$ & $\zeta^{-3}$ & 1 & $\zeta^{-2}$ & $\zeta^{3}$ & 1 \\
\hdashline & & $\bigcirc$ & $\bigcirc$ & & \\
& & &
\end{tabular} & $\zeta \in G_{7}^{\prime}$ \\
\hline
\end{tabular}

Let $V$ be a braided vector space of diagonal type with a basis $\left\{x_{1}, x_{2}\right\}$ and the braiding $c\left(x_{i} \otimes x_{j}\right)=q_{i j} x_{j} \otimes x_{i}$, where $q_{i j} \in \mathbb{k}, i, j \in\{1,2\}$. We choose an abelian group $G$ and the set $\left\{g_{i} \mid g_{i} \in G, i \in\{1,2\}\right\}$ such that the assignments $\delta\left(x_{i}\right)=g_{i} x_{i}, g_{i} . x_{j}=q_{i j} x_{j}$ for $i, j \in\{1,2\}$ define a Yetter-Drinfel'd module structure on $V$ over $\mathbb{k} G$. Let $\mathcal{B}(V)$ denote the Nichols algebra of $V$.

The following theorem determines whether Weyl groupoid $\mathcal{W}\left(\mathbb{k} x_{1}, \mathbb{k} x_{2}\right)$ of $\left(\mathbb{k} x_{1}, \mathbb{k} x_{2}\right)$ is finite in terms of the Dynkin diagram of $V$.

Theorem 5.1. Let $V$ be a two-dimensional braided vector space of diagonal type with the braiding

$$
c\left(x_{i} \otimes x_{j}\right)=q_{i j} x_{j} \otimes x_{i},
$$

where $i, j \in\{1,2\}$ and $\left\{x_{1}, x_{2}\right\}$ is a basis of $V$. Let $M:=\left(\mathbb{k} x_{1}, \mathbb{k} x_{2}\right)$. Assume that the characteristic $p$ of $\mathbb{k}$ is positive. Then the following are equivalent:

(1) $\mathcal{B}(V)$ is decomposable and $\boldsymbol{\Delta}^{[M]}$ is finite,

(2) the Dynkin diagram $\mathcal{D}$ of $V$ appears in Tables 5.1, 5.2, 5.3, 5.4 and 5.5, if $p=2, p=3$, $p=5, p=7$ and $p>7$, respectively,

(3) $M$ admits all refections and $\mathcal{W}(M)$ is finite.

In this case, the row of Table 5.6 containing $\mathcal{D}$ consists precisely of the Dynkin diagrams of the points of $\mathcal{C}_{s}(M)$. Further, the corresponding row of Table 5.7 contains the exchange graph of $\mathcal{C}_{s}(M)$. 
Table 5.2. Dynkin diagrams in characteristic $p=3$.

\begin{tabular}{|c|c|c|}
\hline & Dynkin diagrams & fixed parameters \\
\hline 1 & $\begin{array}{rr}q & r \\
\bigcirc & \bigcirc \\
\end{array}$ & $q, r \in \mathbb{k}^{*}$ \\
\hline 2 & $\stackrel{q}{q} q^{-1} \quad{ }^{-1}$ & $q \in \mathbb{k}^{*} \backslash\{1\}$ \\
\hline 3 & $\begin{array}{cccccc}q & q^{-1} & -1 & -1 & q & -1 \\
0 & & \bigcirc & \bigcirc & & \bigcirc \\
\end{array}$ & $q \in \mathbb{k}^{*} \backslash\{-1,1\}$ \\
\hline 4 & $\begin{array}{lrr}q & q^{-2} & q^{2} \\
\bigcirc & & \bigcirc \\
\end{array}$ & $q \in \mathbb{R}^{*} \backslash\{-1,1\}$ \\
\hline 5 & 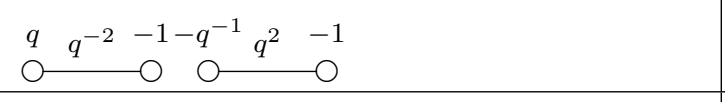 & $q \in \mathbb{k}^{*} \backslash\{-1,1\}, q \notin G_{4}^{\prime}$ \\
\hline $6^{\prime}$ & \begin{tabular}{cccccc}
1 & $q$ & $q^{-1}$ & 1 & $q^{-1}$ & $q$ \\
$\bigcirc$ & & $\bigcirc$ & $\bigcirc$ & & \\
\hdashline
\end{tabular} & $q \in \mathbb{k}^{*} \backslash\{1,-1\}$ \\
\hline $6^{\prime \prime \prime}$ & $\begin{array}{ccc}1 & -1 & -1 \\
0 & - & 0\end{array}$ & \\
\hline $9^{\prime}$ & 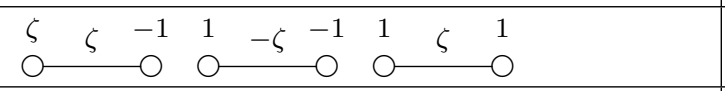 & $\zeta \in G_{4}^{\prime}$ \\
\hline 11 & \begin{tabular}{lrr}
$q$ & $q^{-3}$ & $q^{3}$ \\
\hdashline
\end{tabular} & $q \in \mathbb{k}^{*} \backslash\{-1,1\}$ \\
\hline 12 & 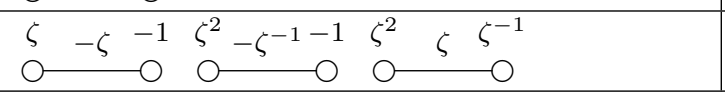 & $\zeta \in G_{8}^{\prime}$ \\
\hline $13^{\prime}$ & 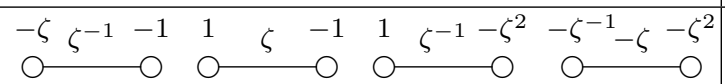 & $\zeta \in G_{8}^{\prime}$ \\
\hline 14 & $\zeta \zeta^{2} \quad-1-\zeta^{-2} \zeta^{-2}-1$ & $\zeta \in G_{5}^{\prime}$ \\
\hline 15 & 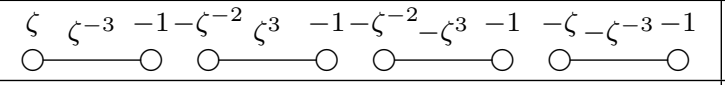 & $\zeta \in G_{20}^{\prime}$ \\
\hline $16^{\prime}$ & 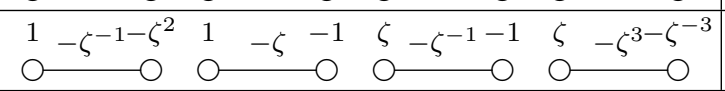 & $\zeta \in G_{5}^{\prime}$ \\
\hline 17 & $\overbrace{}^{-\zeta}-\zeta^{-3}-1-\zeta^{-2}-\zeta^{3}-1$ & $\zeta \in G_{7}^{\prime}$ \\
\hline
\end{tabular}

Remark 5.2. In order to illustrate the exchange graphs of the semi-Cartan graph $\mathcal{C}_{s}(M)$ in Theorem 5.1, we use the following notation in Tables 5.1-5.7.

- In row $n$ of Tables 5.1-5.6 let $\mathcal{D}_{n l}$ be the $l$-th Dynkin diagram for all $l \geq 1$. Since the exchange graph of the semi-Cartan graph is labeled, we write $\tau \mathcal{D}_{n l}$ for the graph $\mathcal{D}_{n l}$ where the two vertices of $\mathcal{D}_{n l}$ change the positions.

- We also use the notation $(2,1,6,1,2,3)^{2}=(2,1,6,1,2,3,2,1,6,1,2,3)$ and $(3,1,5,1)^{3}=$ $(3,1,5,1,3,1,5,1,3,1,5,1)$ in Table 5.7.

Proof. $(1) \Rightarrow(3)$. Since $\mathcal{B}(V)$ is decomposable and $\boldsymbol{\Delta}^{[M]}$ is finite, we obtain that $M$ admits all reflections and $\mathcal{R}(M)=\left(\mathcal{C}(M),\left(\boldsymbol{\Delta}^{[X]}\right)_{[X] \in \mathcal{X}_{2}(M)}\right)$ is a root system of type $\mathcal{C}(M)$ by [16, Corollary 6.12]. Then $\mathcal{W}(M)$ is finite by [16, Lemma 5.1] since $\boldsymbol{\Delta}^{[M]}$ is finite.

$(3) \Rightarrow(1)$. Since $M$ admits all refections and $\mathcal{W}(M)$ is finite, the set $\boldsymbol{\Delta}^{[M]}$ is finite by [16, Lemma 5.1]. Moreover $\mathcal{B}(M)$ is decomposable by [17, Corollary 6.16].

$(2) \Rightarrow(3)$. By Lemma 3.3 one obtains that $M$ is $i$-finite for all $i \in I$. For $i \in I$, one can determine the Dynkin diagram of $R_{i}(M)$ by Lemma 3.4. One observes that it appears in the same row of Table 5.6 as $\mathcal{D}$. Doing the same for all the Dynkin diagrams in the same row of $\mathcal{D}$ implies that $M$ admits all reflections. Hence $\mathcal{C}_{s}(M)$ is well-defined by Proposition 3.6. Now, we identify the objects of $\mathcal{C}_{s}(M)$ with their Dynkin diagrams. 
Table 5.3. Dynkin diagrams in characteristic $p=5$.

\begin{tabular}{|c|c|c|}
\hline & Dynkin diagrams & fixed parameters \\
\hline 1 & $\begin{array}{rr}q & r \\
\bigcirc & \bigcirc \\
\end{array}$ & $q, r \in \mathbb{k}^{*}$ \\
\hline 2 & $\bigcirc^{q} q^{-1} \quad q$ & $q \in \mathbb{k}^{*} \backslash\{1\}$ \\
\hline 3 & $\begin{array}{cccccc}q & q^{-1} & -1 & -1 & q & -1 \\
\bigcirc & & \bigcirc & \bigcirc & & \bigcirc \\
\end{array}$ & $q \in \mathbb{k}^{*} \backslash\{-1,1\}$ \\
\hline 4 & \begin{tabular}{ccc}
$q$ & $q^{-2}$ & $q^{2}$ \\
\hdashline & $\bigcirc$ \\
\end{tabular} & $q \in \mathbb{k}^{*} \backslash\{-1,1\}$ \\
\hline 5 & 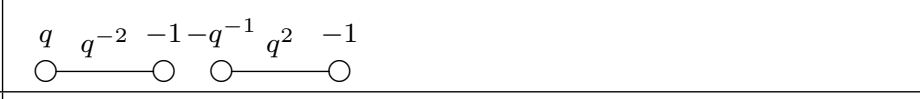 & $q \in \mathbb{k}^{*} \backslash\{-1,1\}, q \notin G_{4}^{\prime}$ \\
\hline 6 & $\bigcirc^{\zeta} q^{-1} \quad q \quad \zeta \quad \zeta^{-1} q \zeta q^{-1}$ & $\begin{array}{l}\zeta \in G_{3}^{\prime}, q \zeta \neq-1 \\
q \in \mathbb{k}^{*} \backslash\left\{1, \zeta, \zeta^{2}\right\}\end{array}$ \\
\hline $6^{\prime \prime}$ & $\zeta-\zeta^{-\zeta^{-1}}$ & $\zeta \in G_{3}^{\prime}$ \\
\hline 7 & $\zeta-\zeta \quad-1 \zeta^{-1}-\zeta^{-1}-1$ & $\zeta \in G_{3}^{\prime}$ \\
\hline 8 & 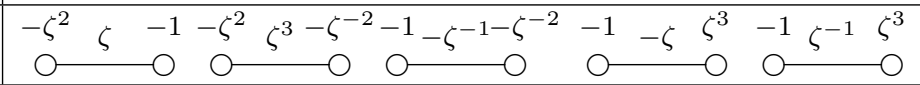 & $\zeta \in G_{12}^{\prime}$ \\
\hline 9 & 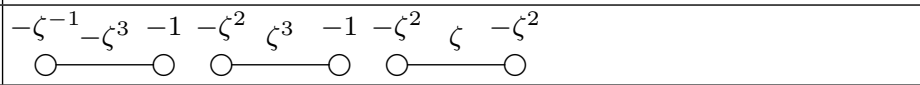 & $\zeta \in G_{12}^{\prime}$ \\
\hline 10 & 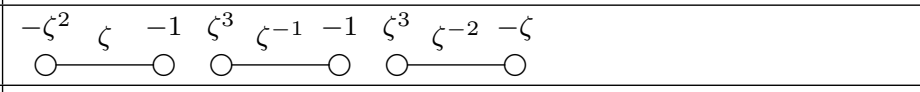 & $\zeta \in G_{9}^{\prime}$ \\
\hline 11 & \begin{tabular}{lll}
$q$ & $q^{-3}$ & $q^{3}$ \\
\hdashline & $\bigcirc$
\end{tabular} & $q \in \mathbb{k}^{*} \backslash\{-1,1\}, q \notin G_{3}^{\prime}$ \\
\hline 12 & 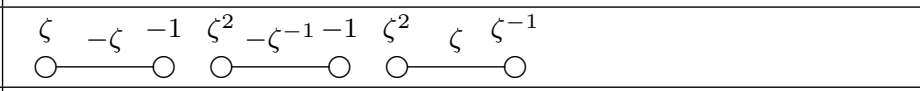 & $\zeta \in G_{8}^{\prime}$ \\
\hline 13 & 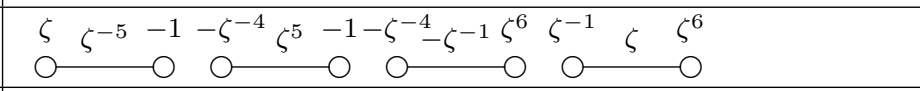 & $\zeta \in G_{24}^{\prime}$ \\
\hline $15^{\prime}$ & 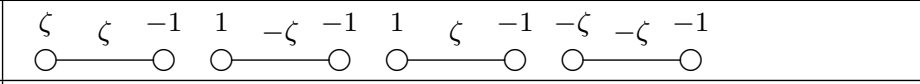 & $\zeta \in G_{4}^{\prime}$ \\
\hline $16^{\prime \prime}$ & $\begin{array}{cccccccccc}\zeta^{2} & -1 & -\zeta & \zeta^{2} & -\zeta & -1 & 1 & -\zeta^{-1}-1 & 1 & -\zeta^{-\zeta^{-1}} \\
\bigcirc & & 0 & \bigcirc & & \circ & \circ & \bigcirc & \bigcirc & \bigcirc\end{array}$ & $\zeta \in G_{3}^{\prime}$ \\
\hline 17 & $\begin{array}{ccc}-\zeta & -\zeta^{-3}-1 & -\zeta^{-2}-\zeta^{3}-1 \\
0 & 0 & 0\end{array}$ & $\zeta \in G_{7}^{\prime}$ \\
\hline
\end{tabular}

Assume that $\mathcal{D}$ appears in row $r$ of one of Tables 5.1-5.6. Then by the above calculations, the exchange graph of $\mathcal{C}_{s}(M)$ appears in row $r$ of Table 5.7. Then we calculate the smallest integer $n$ with $\left(r_{2} r_{1}\right)^{n}(\mathcal{D})=\mathcal{D}$ and we observe that it appears in the third column of row $r$ of Table 5.7.

Then we compute the characteristic sequence $\left(c_{k}\right)_{k \geq 1}$ with respect to the first Dynkin diagram in row $r$ and the label 1 . We observe that $\left(c_{k}\right)_{k \geq 1}$ is the infinite power of the sequence in the fifth column of row $r$ of Table 5.7. Further, we get the numbers $l=6 n-\sum_{i=1}^{2 n} c_{i}$. They appear in the fourth column of Table 5.7. One checks that $l \mid 12$ and $\left(c_{1}, c_{2}, \ldots, c_{12 n / l}\right) \in \mathcal{A}^{+}$by Corollary $4.5(2)$. The detailed calculations are skipped at this point here. Then Theorem 4.15 implies that $\mathcal{C}_{s}(M)$ is a finite Cartan graph. Hence $\mathcal{C}(M)$ is a finite Cartan graph and $\mathcal{W}(M)$ is a finite Weyl groupoid by [16, Lemma 5.1].

$(3) \Rightarrow(2)$. Since $M$ admits all reflections, the tuple

$$
\mathcal{C}(M)=\left\{I, \mathcal{X}_{2}(M),\left(r_{i}\right)_{i \in I},\left(A^{X}\right)_{X \in \mathcal{X}_{2}(M)}\right\}
$$


Table 5.4. Dynkin diagrams in characteristic $p=7$.

\begin{tabular}{|c|c|c|}
\hline & Dynkin diagrams & fixed parameters \\
\hline 1 & $\begin{array}{cc}q & r \\
0 & \bigcirc\end{array}$ & $q, r \in \mathbb{k}^{*}$ \\
\hline 2 & $\begin{array}{c}q \\
\end{array} q^{-1} \quad q$ & $q \in \mathbb{k}^{*} \backslash\{1\}$ \\
\hline 3 & \begin{tabular}{cccccc}
$q$ & $q^{-1}$ & -1 & -1 & $q$ & -1 \\
\hdashline & $\bigcirc$ & $\bigcirc$ & & $\bigcirc$ \\
\end{tabular} & $q \in \mathbb{k}^{*} \backslash\{-1,1\}$ \\
\hline 4 & \begin{tabular}{lrr}
$q$ & $q^{-2}$ & $q^{2}$ \\
\hdashline & & $\bigcirc$ \\
\end{tabular} & $q \in \mathbb{k}^{*} \backslash\{-1,1\}$ \\
\hline 5 & 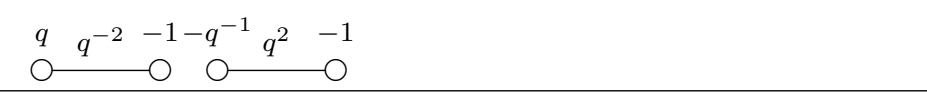 & $q \in \mathbb{k}^{*} \backslash\{-1,1\}, q \notin G_{4}^{\prime}$ \\
\hline 6 & 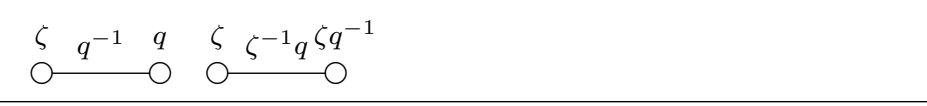 & $\begin{array}{l}\zeta \in G_{3}^{\prime}, q \zeta \neq-1 \\
q \in \mathbb{k}^{*} \backslash\left\{1, \zeta, \zeta^{2}\right\}\end{array}$ \\
\hline $6^{\prime \prime}$ & $\zeta \zeta^{-\zeta^{-1}}$ & $\zeta \in G_{3}^{\prime}$ \\
\hline 7 & 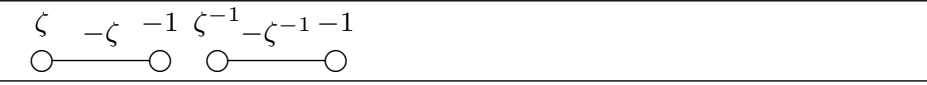 & $\zeta \in G_{3}^{\prime}$ \\
\hline 8 & 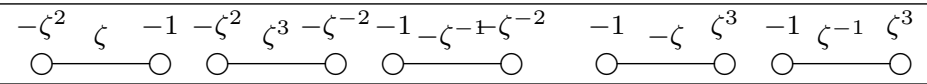 & $\zeta \in G_{12}^{\prime}$ \\
\hline 9 & 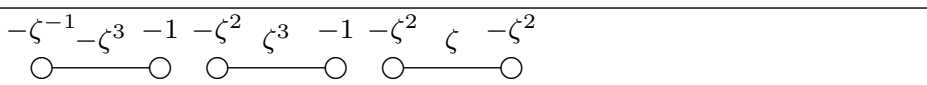 & $\zeta \in G_{12}^{\prime}$ \\
\hline 10 & 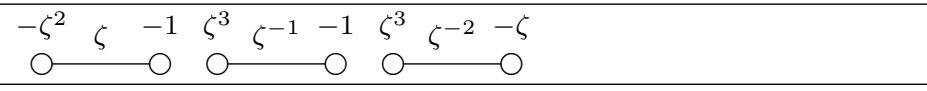 & $\zeta \in G_{9}^{\prime}$ \\
\hline 11 & \begin{tabular}{lll}
$q$ & $q^{-3} \quad q^{3}$ \\
\hdashline
\end{tabular} & $q \in \mathbb{k}^{*} \backslash\{-1,1\}, q \notin G_{3}^{\prime}$ \\
\hline 12 & 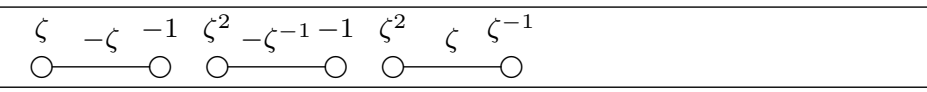 & $\zeta \in G_{8}^{\prime}$ \\
\hline 13 & $\stackrel{\zeta}{\zeta} \zeta^{-5}-1-\zeta^{-4} \zeta^{5}-1-\zeta^{-4}-\zeta^{-1} \zeta^{6} \zeta^{-1} \quad \zeta \quad \zeta^{6}$ & $\zeta \in G_{24}^{\prime}$ \\
\hline 14 & $\zeta \zeta^{2} \stackrel{-1-\zeta^{-2} \zeta^{-2}-1}{\circ} \bigcirc$ & $\zeta \in G_{5}^{\prime}$ \\
\hline 15 & 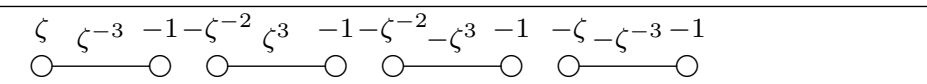 & $\zeta \in G_{20}^{\prime}$ \\
\hline 16 & 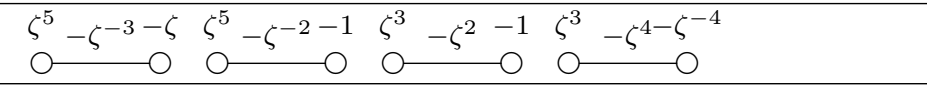 & $\zeta \in G_{15}^{\prime}$ \\
\hline 18 & 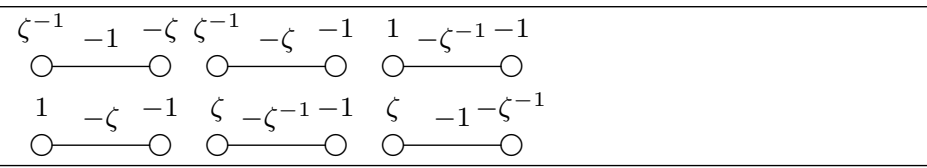 & $\zeta \in G_{3}^{\prime}$ \\
\hline
\end{tabular}

defined in Theorem 3.1 is a semi-Cartan graph. In particular,

$$
\mathcal{X}_{2}(M)=\left\{\left[R_{i_{1}} \cdots R_{i_{n}}(M)\right] \in \mathcal{X}_{2} \mid n \in \mathbb{N}_{0}, i_{1}, \ldots, i_{n} \in I\right\}
$$

and $R_{i}(M)=\left(R_{i}(M)_{j}\right)_{j \in I}$ is defined by equation (3.2). Moreover, $A^{X}=\left(a_{i j}^{X}\right)_{i, j \in I}$ for all $X \in \mathcal{X}_{2}(M)$, where

$$
-a_{i j}^{X}=\min \left\{m \in \mathbb{N}_{0} \mid(m+1) q_{i i}^{\prime}\left(q_{i i}^{\prime}{ }^{m} q_{i j}^{\prime} q_{j i}^{\prime}-1\right)=0\right\}
$$

by Lemma 3.2 and $\left(q_{i j}^{\prime}\right)_{i, j \in I}$ is the braiding matrix of $X$. Then $\Delta^{[M]}$ is finite since $\mathcal{W}(M)$ is finite. Hence all roots are real by [10, Proposition 2.12]. Then $\mathcal{C}(M)$ is a finite Cartan graph by Theorem 3.1. Hence $\mathcal{C}_{s}(M)$ is a finite Cartan graph by Proposition 3.6. We can apply Theorem 4.15 to $\mathcal{C}_{s}(M)$. 
Table 5.5. Dynkin diagrams in characteristic $p>7$.

\begin{tabular}{|c|c|c|}
\hline & Dynkin diagrams & fixed parameters \\
\hline 1 & $\begin{array}{rr}q & r \\
\bigcirc & \bigcirc \\
\end{array}$ & $q, r \in \mathbb{k}^{*}$ \\
\hline 2 & \begin{tabular}{ccc}
$q$ & $q^{-1}$ & $q$ \\
\hdashline & & $\bigcirc$ \\
\end{tabular} & $q \in \mathbb{k}^{*} \backslash\{1\}$ \\
\hline 3 & $\begin{array}{cccccc}q & q^{-1} & -1 & -1 & q & -1 \\
\bigcirc & & \bigcirc & \bigcirc & & \bigcirc \\
\end{array}$ & $q \in \mathbb{k}^{*} \backslash\{-1,1\}$ \\
\hline 4 & 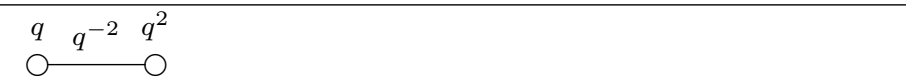 & $q \in \mathbb{k}^{*} \backslash\{-1,1\}$ \\
\hline 5 & 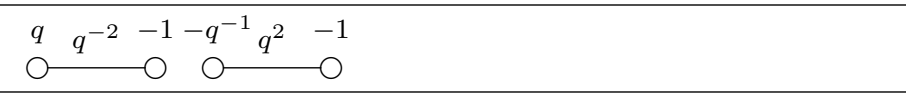 & $\begin{array}{l}q \in \mathbb{k}^{*} \backslash\{-1,1\} \\
q \notin G_{4}^{\prime}\end{array}$ \\
\hline 6 & $\begin{array}{lllll}\zeta & q^{-1} & q & \zeta & \zeta^{-1} q \zeta q^{-1} \\
\bigcirc & \bigcirc & \bigcirc & \bigcirc\end{array}$ & $\begin{array}{l}\zeta \in G_{3}^{\prime}, q \zeta \neq-1 \\
q \in \mathbb{k}^{*} \backslash\left\{1, \zeta, \zeta^{2}\right\}\end{array}$ \\
\hline $6^{\prime \prime}$ & $\overbrace{-}^{-} \zeta^{-\zeta^{-1}}$ & $\zeta \in G_{3}^{\prime}$ \\
\hline 7 & 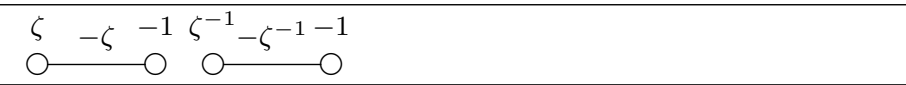 & $\zeta \in G_{3}^{\prime}$ \\
\hline 8 & 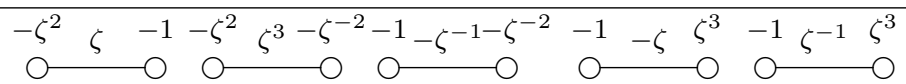 & $\zeta \in G_{12}^{\prime}$ \\
\hline 9 & $\begin{array}{cccccccc}-\zeta^{-1}-\zeta^{3} & -1 & -\zeta^{2} & \zeta^{3} & -1 & -\zeta^{2} & \zeta & -\zeta^{2} \\
0 & 0 & 0 & 0 & 0 & 0 & 0\end{array}$ & $\zeta \in G_{12}^{\prime}$ \\
\hline 10 & 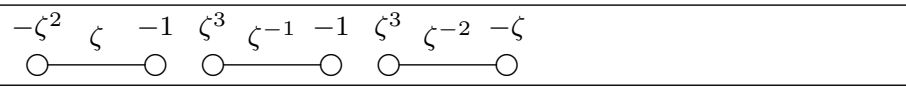 & $\zeta \in G_{9}^{\prime}$ \\
\hline 11 & \begin{tabular}{lrr}
$q$ & $q^{-3}$ & $q^{3}$ \\
\hdashline & & $\bigcirc$ \\
\end{tabular} & $\begin{array}{l}q \in \mathbb{k}^{*} \backslash\{-1,1\} \\
q \notin G_{3}^{\prime}\end{array}$ \\
\hline 12 & 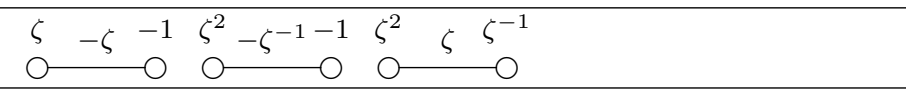 & $\zeta \in G_{8}^{\prime}$ \\
\hline 13 & 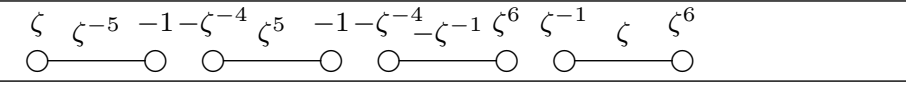 & $\zeta \in G_{24}^{\prime}$ \\
\hline 14 & 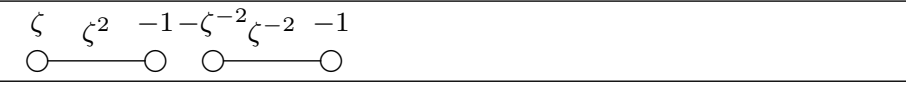 & $\zeta \in G_{5}^{\prime}$ \\
\hline 15 & $\zeta \zeta^{-3}-1-\zeta^{-2} \zeta^{3} \quad-1-\zeta^{-2}-\zeta^{3}-1 \quad-\zeta-\zeta^{-3-1}$ & $\zeta \in G_{20}^{\prime}$ \\
\hline 16 & 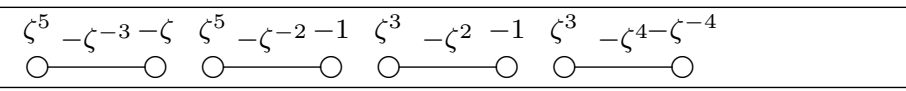 & $\zeta \in G_{15}^{\prime}$ \\
\hline 17 & $\begin{array}{ccc}-\zeta & -\zeta^{-3}-1-\zeta^{-2}-\zeta^{3} & -1 \\
\bigcirc & \bigcirc & \bigcirc\end{array}$ & $\zeta \in G_{7}^{\prime}$ \\
\hline
\end{tabular}

By the implication $(2) \Rightarrow(3)$, it is enough to prove that the Dynkin diagram of at least one point in $\mathcal{C}_{s}(M)$ is contained in Table 5.6.

Set $X=[M]_{s}$ and $m=t_{12}^{X}$. Let $\left(c_{k}\right)_{k \geq 1}$ be the characteristic sequence of $\mathcal{C}_{s}(M)$ with respect to $X$ and the label 1. Then we have $\left(c_{1}, c_{2}, \ldots, c_{m}\right) \in \mathcal{A}^{+}$and $\left(c_{k}\right)_{k \geq 1}=\left(c_{1}, c_{2}, \ldots, c_{m}\right)^{\infty}$ by Theorem 4.15 .

If $m=2$ then $\left(c_{1}, c_{2}\right)=(0,0)$. Hence $a_{12}^{X}=a_{21}^{X}=0$. Then $q_{12} q_{21}=1$ and $\mathcal{D}=\mathcal{D}_{11}$.

If $m>2$, by Theorem 4.6, one of $(1,1),(1,2, a),(2,1, b),(1,3,1, b)$ or their transpose, where $1 \leq a \leq 3$ and $3 \leq b \leq 5$, is a subsequence of $\left(c_{1}, c_{2}, \ldots, c_{m}\right)$. Let $n$ be the smallest integer with $\left(r_{2} r_{1}\right)^{n}(X)=X$. Then $n \mid m$ by Theorem 4.15 . Since $c_{m+k}=c_{k}$ for all $k \in \mathbb{N}$, we have the freedom to assume any position in $\left(c_{k}\right)_{k \geq 1}$, where any of these subsequences is starting. Let $c_{0}=c_{m}$ and $q_{0}=q_{12} q_{21}$.

We may assume that $i=1, j=2$, but change the labels if necessary. Next we proceed case by case: 
Table 5.6. The Dynkin diagrams in Theorem 5.1.

\begin{tabular}{|c|c|c|c|}
\hline & Dynkin diagrams & fixed parameters & $\operatorname{char} \mathbb{k}$ \\
\hline 1 & $\begin{array}{rr}q & r \\
\bigcirc & \bigcirc\end{array}$ & $q, r \in \mathbb{k}^{*}$ & \\
\hline 2 & \begin{tabular}{ccc}
$q$ & $q^{-1}$ & $q$ \\
\hdashline & $\bigcirc$
\end{tabular} & $q \in \mathbb{k}^{*} \backslash\{1\}$ & \\
\hline 3 & $\begin{array}{cccccc}q & q^{-1} & -1 & -1 & q & -1 \\
\bigcirc & \bigcirc & \bigcirc & \bigcirc\end{array}$ & $q \in \mathbb{k}^{*} \backslash\{-1,1\}$ & \\
\hline 4 & $\begin{array}{lll}q & q^{-2} & q^{2} \\
\bigcirc & \bigcirc\end{array}$ & $q \in \mathbb{k}^{*} \backslash\{-1,1\}$ & \\
\hline 5 & $\begin{array}{cccccc}q & q^{-2} & -1 & -q^{-1} & q^{2} & -1 \\
\bigcirc & 0 & \bigcirc & & \bigcirc\end{array}$ & $\begin{array}{l}q \in \mathbb{k}^{*} \backslash\{-1,1\} \\
q \notin G_{4}^{\prime}\end{array}$ & \\
\hline 6 & 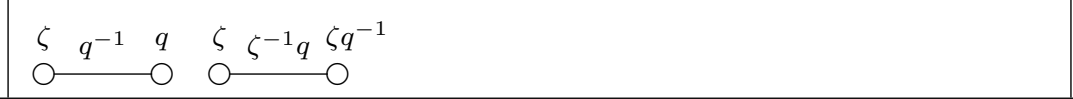 & $\left|\begin{array}{l}\zeta \in G_{3}^{\prime}, q \zeta \neq-1 \\
q \in \mathbb{k}^{*} \backslash\left\{1, \zeta, \zeta^{2}\right\}\end{array}\right|$ & $p \neq 3$ \\
\hline $6^{\prime}$ & 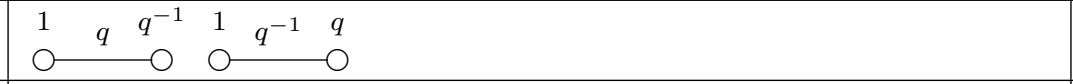 & $q \in \mathbb{k}^{*} \backslash\{1,-1\}$ & $p=3$ \\
\hline $6^{\prime \prime}$ & $\zeta-\zeta^{-\zeta^{-1}}$ & $\zeta \in G_{3}^{\prime}$ & $p \neq 2,3$ \\
\hline $6^{\prime \prime \prime}$ & $\begin{array}{ccc}1 & -1 & -1 \\
0 & - & 0\end{array}$ & & $p=3$ \\
\hline 7 & $\begin{array}{cccc}\zeta & -\zeta & -1 & \zeta^{-1}-\zeta^{-1}-1 \\
\bigcirc & \bigcirc & \bigcirc & \bigcirc\end{array}$ & $\zeta \in G_{3}^{\prime}$ & $p \neq 3$ \\
\hline 8 & 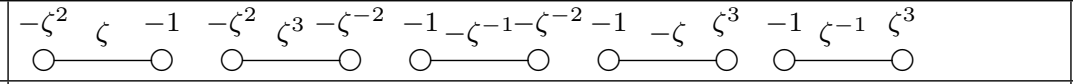 & $\zeta \in G_{12}^{\prime}$ & $p \neq 2,3$ \\
\hline 9 & 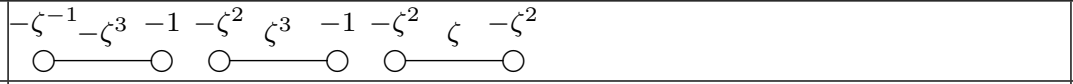 & $\zeta \in G_{12}^{\prime}$ & $p \neq 2,3$ \\
\hline $9^{\prime}$ & $\begin{array}{ccccccccc}\zeta & \zeta & -1 & 1 & -\zeta & -1 & 1 & \zeta & 1 \\
\bigcirc & 0 & \bigcirc & & \bigcirc & \bigcirc & & \bigcirc \\
\end{array}$ & $\zeta \in G_{4}^{\prime}$ & $p=3$ \\
\hline 10 & $\begin{array}{ccccccccc}-\zeta^{2} & & -1 & \zeta^{3} & \zeta^{-1} & -1 & \zeta^{3} & \zeta^{-2} & -\zeta \\
0 & & 0 & 0 & & 0 & 0 & & 0\end{array}$ & $\zeta \in G_{9}^{\prime}$ & $p \neq 3$ \\
\hline 11 & \begin{tabular}{lrr}
$q$ & $q^{-3}$ & $q^{3}$ \\
\hdashline & & $\bigcirc$ \\
\end{tabular} & $\begin{array}{l}q \in \mathbb{k}^{*} \backslash\{-1,1\} \\
q \notin G_{3}^{\prime}\end{array}$ & \\
\hline 12 & 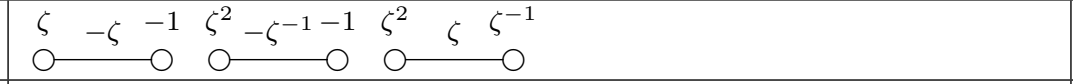 & $\zeta \in G_{8}^{\prime}$ & $p \neq 2$ \\
\hline 13 & 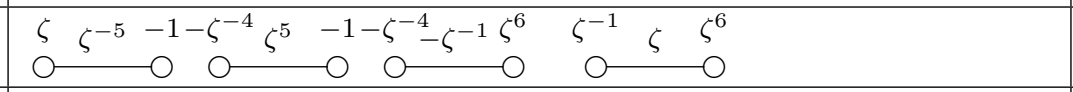 & $\zeta \in G_{24}^{\prime}$ & $p \neq 2,3$ \\
\hline $13^{\prime}$ & 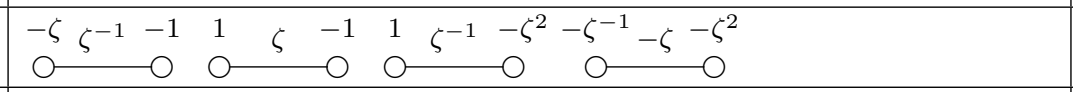 & $\zeta \in G_{8}^{\prime}$ & $p=3$ \\
\hline 14 & 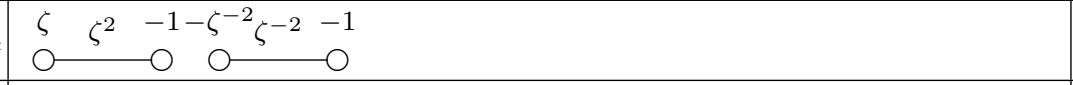 & $\zeta \in G_{5}^{\prime}$ & $p \neq 5$ \\
\hline 15 & 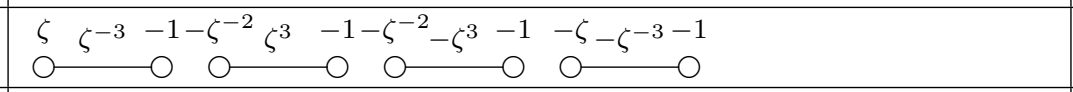 & $\zeta \in G_{20}^{\prime}$ & $p \neq 2,5$ \\
\hline $15^{\prime}$ & $\begin{array}{cccccccccccc}\zeta & \zeta & -1 & 1 & -\zeta & -1 & 1 & \zeta & -1 & -\zeta & -\zeta & -1 \\
0 & 0 & \bigcirc & & 0 & \bigcirc & & 0 & 0 & & 0\end{array}$ & $\zeta \in G_{4}^{\prime}$ & $p=5$ \\
\hline 16 & 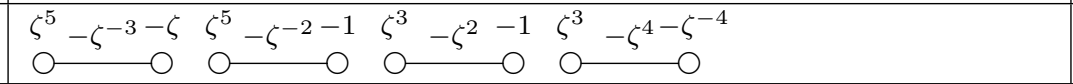 & $\zeta \in G_{15}^{\prime}$ & $p \neq 3,5$ \\
\hline $16^{\prime}$ & 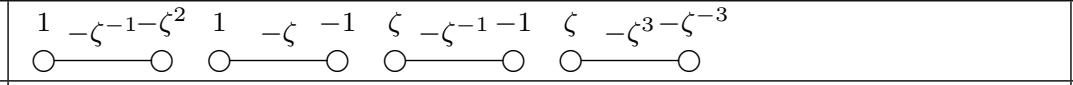 & $\zeta \in G_{5}^{\prime}$ & $p=3$ \\
\hline $16^{\prime \prime}$ & 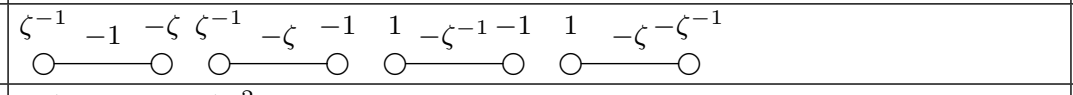 & $\zeta \in G_{3}^{\prime}$ & $p=5$ \\
\hline 17 & $\begin{array}{ccc}-\zeta & -\zeta^{-3}-1-\zeta^{-2}-\zeta^{3}-1 \\
0 & 0 & 0\end{array}$ & $\zeta \in G_{7}^{\prime}$ & $p \neq 7$ \\
\hline 18 & 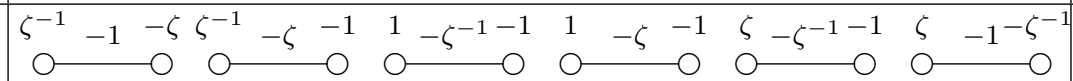 & $\zeta \in G_{3}^{\prime}$ & $p=7$ \\
\hline
\end{tabular}


Table 5.7. The exchange graphs of $\mathcal{C}_{s}(M)$ in Theorem 5.1.

\begin{tabular}{|c|c|c|c|c|c|}
\hline & exchange graphs & $n$ & $l$ & sequences in $\mathcal{A}^{+}$ & char $\mathbb{k}$ \\
\hline 1 & $\mathcal{D}_{11}$ & 1 & 6 & $(0,0)$ & \\
\hline 2 & $\mathcal{D}_{21}$ & 1 & 4 & $(1,1,1)$ & \\
\hline 3 & $\mathcal{D}_{31} \stackrel{2}{-} \mathcal{D}_{32} \stackrel{1}{-} \tau \mathcal{D}_{31}$ & 3 & 12 & $(1,1,1)$ & \\
\hline 4 & $\mathcal{D}_{41}$ & 1 & 3 & $(2,1,2,1)$ & \\
\hline 5 & $\mathcal{D}_{51} \stackrel{2}{-} \mathcal{D}_{52}$ & 2 & 6 & $(2,1,2,1)$ & \\
\hline 6 & $\mathcal{D}_{61} \stackrel{1}{-} \mathcal{D}_{62}$ & 2 & 6 & $(2,1,2,1)$ & $p \neq 3$ \\
\hline $6^{\prime}$ & $\mathcal{D}_{6^{\prime}, 1}{ }^{1} \mathcal{D}_{6^{\prime}, 2}$ & 2 & 6 & $(2,1,2,1)$ & $p=3$ \\
\hline $6^{\prime \prime}$ & $\mathcal{D}_{6^{\prime \prime}, 1}$ & 1 & 3 & $(2,1,2,1)$ & $p \neq 2,3$ \\
\hline $6^{\prime \prime \prime}$ & $\mathcal{D}_{6^{\prime \prime \prime}, 1}$ & 1 & 3 & $(2,1,2,1)$ & $p=3$ \\
\hline 7 & $\mathcal{D}_{71} \stackrel{2}{-} \mathcal{D}_{72}$ & 2 & 6 & $(2,1,2,1)$ & $p \neq 3$ \\
\hline 8 & 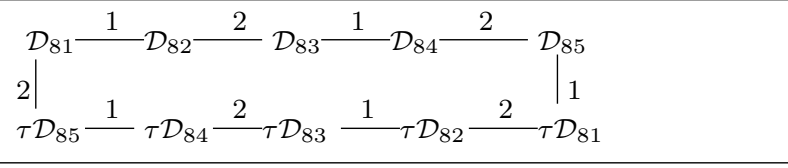 & 5 & 12 & $(2,2,1,3,1)$ & $p \neq 2,3$ \\
\hline 9 & $\mathcal{D}_{91} \stackrel{2}{-} \mathcal{D}_{92} \stackrel{1}{\longrightarrow} \mathcal{D}_{93} \stackrel{2}{\longrightarrow} \mathcal{D}_{92} \stackrel{1}{\tau} \tau \mathcal{D}_{91}$ & 5 & 12 & $(3,1,2,2,1)$ & $p \neq 2,3$ \\
\hline $9^{\prime}$ & $\mathcal{D}_{9^{\prime}, 1} \stackrel{2}{-} \mathcal{D}_{9^{\prime}, 2} \stackrel{1}{-} \mathcal{D}_{9^{\prime}, 3} \stackrel{2}{\longleftarrow} \tau \mathcal{D}_{9^{\prime}, 2} \frac{1}{\tau \mathcal{D}_{9^{\prime}, 1}}$ & 5 & 12 & $(3,1,2,2,1)$ & $p=3$ \\
\hline 10 & $\mathcal{D}_{10, \Gamma} \stackrel{2}{=} \mathcal{D}_{10,2} \stackrel{1}{-} \mathcal{D}_{10,3}$ & 3 & 6 & $(4,1,2,2,2,1)$ & $p \neq 3$ \\
\hline 11 & $\mathcal{D}_{11,1}$ & 1 & 2 & $(3,1,3,1,3,1)$ & \\
\hline 12 & $\mathcal{D}_{12,1} \stackrel{2}{-} \mathcal{D}_{12,2} \stackrel{1}{-} \mathcal{D}_{12,3}$ & 3 & 6 & $(3,1,3,1,3,1)$ & $p \neq 2$ \\
\hline 13 & $\mathcal{D}_{13,1}{ }^{2} \mathcal{D}_{13,2}{ }^{1} \mathcal{D}_{13,3}{ }^{2} \mathcal{D}_{13,4}$ & 4 & 6 & $(5,1,2,3,1,3,2,1)$ & $p \neq 2,3$ \\
\hline $13^{\prime}$ & $\mathcal{D}_{13^{\prime}, \frac{2}{1}} \mathcal{D}_{13^{\prime}, \frac{1}{2}} \mathcal{D}_{13^{\prime}, 3} \frac{2}{\mathcal{D}_{13^{\prime}, 4}}$ & 4 & 6 & $(5,1,2,3,1,3,2,1)$ & $p=3$ \\
\hline 14 & $\mathcal{D}_{14,1}{ }^{2} \mathcal{D}_{14,2}$ & 2 & 3 & $(3,1,4,1,3,1,4,1)$ & $p \neq 5$ \\
\hline 15 & $\mathcal{D}_{15,1} \stackrel{2}{=} \mathcal{D}_{15,2} \stackrel{1}{-} \mathcal{D}_{15,3} \stackrel{2}{=} \mathcal{D}_{15,4}$ & 4 & 6 & $(3,1,4,1,3,1,4,1)$ & $p \neq 2,5$ \\
\hline $15^{\prime}$ & $\mathcal{D}_{15^{\prime}, 1} \stackrel{2}{-} \mathcal{D}_{15^{\prime}, 2} \frac{1}{\mathcal{D}_{15^{\prime}, 3}}{ }^{2} \mathcal{D}_{15^{\prime}, 4}$ & 4 & 6 & $(3,1,4,1,3,1,4,1)$ & $p=5$ \\
\hline 16 & $\mathcal{D}_{16,1} \stackrel{1}{\stackrel{1}{-}} \mathcal{D}_{16,2} \stackrel{2}{-} \mathcal{D}_{16,3}{ }^{1} \mathcal{D}_{16,4}$ & 4 & 6 & $(2,1,4,1,4,1,2,3)$ & $p \neq 3,5$ \\
\hline $16^{\prime}$ & $\mathcal{D}_{16^{\prime}, \frac{1}{1}} \mathcal{D}_{16^{\prime}, 2} \stackrel{2}{-} \mathcal{D}_{16^{\prime}, 3} \stackrel{1}{-} \mathcal{D}_{16^{\prime}, 4}$ & 4 & 6 & $(2,1,4,1,4,1,2,3)$ & $p=3$ \\
\hline $16^{\prime \prime}$ & $\mathcal{D}_{16^{\prime \prime}, 1} \stackrel{1}{-} \mathcal{D}_{16^{\prime \prime}, 2} \stackrel{2}{-} \mathcal{D}_{16^{\prime \prime}, 3} \frac{1}{-} \mathcal{D}_{16^{\prime \prime}, 4}$ & 4 & 6 & $(2,1,4,1,4,1,2,3)$ & $p=5$ \\
\hline 17 & $\mathcal{D}_{17,1} \stackrel{2}{\longrightarrow} \mathcal{D}_{17,2}$ & 2 & 2 & $(3,1,5,1)^{3}$ & $p \neq 7$ \\
\hline 18 & $\mathcal{D}_{18,1} \stackrel{1}{-} \mathcal{D}_{18,2} \stackrel{2}{-} \mathcal{D}_{18,3} \stackrel{1}{-} \mathcal{D}_{18,4} \stackrel{2}{-} \mathcal{D}_{18,5}{ }^{1} \mathcal{D}_{18,6}$ & 6 & 6 & $(2,1,6,1,2,3)^{2}$ & $p=7$ \\
\hline
\end{tabular}

Step 1. If $c_{0}=c_{1}=1$, then $a_{12}^{X}=a_{21}^{X}=-1$. Hence $q_{0} \neq 1$. We distinguish four cases: 1aa, $1 \mathrm{ab}, 1 \mathrm{ba}$ and $1 \mathrm{bb}$.

Case 1aa. If $q_{11} q_{0}=1$ and $q_{22} q_{0}=1$, then $\mathcal{D}=\mathcal{D}_{21}$.

Case 1ab. If $q_{11} q_{0}=1, q_{22}=-1$, and $q_{22} q_{0} \neq 1$, then $\mathcal{D}=\mathcal{D}_{31}$. 
Case 1ba. If $q_{11}=-1, q_{22} q_{0}=1$, and $q_{11} q_{0} \neq 1$, then $\mathcal{D}=\tau \mathcal{D}_{31}$.

Case 1bb. If $q_{11}=-1, q_{22}=-1$, and $q_{0} \neq-1$, then $\mathcal{D}=\mathcal{D}_{32}$.

Step 2. Assume that $\left(c_{0}, c_{1}, c_{2}\right)=\left(1,2, a^{\prime}\right)$, where $a^{\prime} \in\{1,2,3\}$. Then we obtain that $a_{21}^{X}=-1, a_{12}^{X}=a_{12}^{r_{1}(X)}=-2$, and $a_{21}^{r_{1}(X)}=-a^{\prime}$. We distinguish four cases: 2aa, 2ab, $2 \mathrm{ba}$ and $2 \mathrm{bb}$.

Case 2aa. If $q_{11}^{2} q_{0}=1$ and $q_{22} q_{0}=1$, then $\mathcal{D}=\mathcal{D}_{41}$.

Case 2ab. If $q_{11}^{2} q_{0}=1, q_{22}=-1$, and $q_{22} q_{0} \neq 1$, then $\mathcal{D}=\mathcal{D}_{51}$.

Case 2ba. Assume that $1+q_{11}+q_{11}^{2}=0, q_{22} q_{0}=1$, and $q_{11}^{2} q_{0} \neq 1$. If $p=3$ then $1+q_{11}+q_{11}^{2}=0$ yields $q_{11}=1$. If $q_{22} \neq-1$ then $\mathcal{D}=\mathcal{D}_{6^{\prime}, 1}$ and if $q_{22}=-1$ then $\mathcal{D}=\mathcal{D}_{6^{\prime \prime \prime}, 1}$. Assume that $p \neq 3$. Set $\zeta:=q_{11}$ and $q:=q_{22}$. Then $q_{0}=q^{-1} \notin\left\{1, \zeta^{-1}\right\}$ since $a_{12}^{X}=-2$, and $q_{0} \neq \zeta$ since $q_{11}^{2} q_{0} \neq 1$. Thus $\mathcal{D}=\mathcal{D}_{61}$ or $\mathcal{D}_{6^{\prime \prime}, 1}, p \neq 2$.

Case 2 bb. Consider the last case $1+q_{11}+q_{11}^{2}=0, q_{22}=-1$ and $q_{0} \notin\left\{1,-1, q_{11}, q_{11}^{2}\right\}$.

Case 2bba. If $p=3$ then $q_{11}=1$. Set $q:=q_{0}$. By Lemma 3.4, the Dynkin diagrams of $r_{1}(X)$ and $X$ are

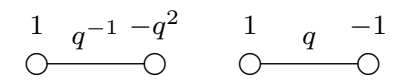

with $q \in \mathbb{k}^{*} \backslash\{-1,1\}$. Then $a_{21}^{r_{1}(X)} \leq-2$ since $\left(-q^{2}\right) q^{-1}=-q \neq 1$, and $-q^{2} \neq-1$.

Case 2bba1. If $p=3$ and $a^{\prime}=-a_{21}^{r_{1}(X)}=2$ then one gets $\left(-q^{2}\right)^{2} q^{-1}=1$ or $1+\left(-q^{2}\right)+$ $\left(-q^{2}\right)^{2}=0$. If $\left(-q^{2}\right)^{2} q^{-1}=1$ then $q=1$, which is a contradiction. Hence $-q^{2}=1$ from the second equation since $p=3$. Then $\mathcal{D}=\mathcal{D}_{9^{\prime}, 2}$.

Case 2bba2. If $p=3$ and $a^{\prime}=-a_{21}^{r_{1}(X)}=3$, then one has $\left(-q^{2}\right)^{3} q^{-1}=1$ or $1+\left(-q^{2}\right)+$ $\left(-q^{2}\right)^{2}+\left(-q^{2}\right)^{3}=0$. The first equation $\left(-q^{2}\right)^{3} q^{-1}=1$ yields $(-q)^{5}=1$, hence $\mathcal{D}=\mathcal{D}_{16^{\prime}, 2}$. If $1+\left(-q^{2}\right)+\left(-q^{2}\right)^{2}+\left(-q^{2}\right)^{3}=0$, then $\left(1-q^{2}\right)\left(1+q^{4}\right)=0$ and hence $q \in G_{8}^{\prime}$. Then $\mathcal{D}=\mathcal{D}_{13^{\prime}, 2}$.

Case 2bbb. We now suppose that $p \neq 3$. Set $\zeta:=q_{11}$ and $q:=q_{0}$. Hence the Dynkin diagram of $r_{1}(X)$ is $\stackrel{\zeta}{\zeta}(\zeta q)^{-1-\zeta q^{2}}$ with $\zeta \in G_{3}^{\prime}, q \in \mathbb{k}^{*} \backslash\left\{1,-1, \zeta, \zeta^{-1}\right\}$. Since $a^{\prime} \in\{1,2,3\}$, we distinguish three cases:

(b1) $p \neq 3, a_{21}^{r_{1}(X)}=1$,

(b2) $p \neq 3, a_{21}^{r_{1}(X)}=2$,

(b3) $p \neq 3, a_{21}^{r_{1}(X)}=3$.

Case 2bbb1. If the condition (b1) holds, then one gets $\left(-\zeta q^{2}\right)(\zeta q)^{-1}=1$ or $1+\left(-\zeta q^{2}\right)=0$. If $\left(-\zeta q^{2}\right)(\zeta q)^{-1}=1$, then $q=-1$, which is a contradiction. If $1+\left(-\zeta q^{2}\right)=0$ then $\zeta^{2}=q^{2}$ and hence $q=-\zeta$. Then $\mathcal{D}=\mathcal{D}_{71}$.

Case 2bbb2. If the condition (b2) holds, then $\left(-\zeta q^{2}\right)^{2}(\zeta q)^{-1}=1$ or $\sum_{i=0}^{2}\left(-\zeta q^{2}\right)^{i}=0$.

Case 2bbb2a. Consider the equation $\left(-\zeta q^{2}\right)^{2}(\zeta q)^{-1}=1$. Then $\zeta q^{3}=1$ and hence $q \in G_{9}^{\prime}$ since $\zeta \in G_{3}^{\prime}$ and $p \neq 3$. Hence $\mathcal{D}=\mathcal{D}_{10,2}$.

Case 2bbb2b. If $\sum_{i=0}^{2}\left(-\zeta q^{2}\right)^{i}=0$, then $-\zeta q^{2} \in\left\{\zeta, \zeta^{-1}\right\}$. Hence $q^{2}=-1$ or $-q^{2}=\zeta$.

Case 2bbb2b1. If $q^{2}=-1$, then $p \neq 2$ and the Dynkin diagram of $X$ is ${ }_{\bigcirc}^{\zeta}{ }_{q}^{-1}$ ith $q \in G_{4}^{\prime}$, $\zeta \in G_{3}^{\prime}$. Set $\eta:=\zeta^{2} q^{-1}$. Then $\eta \in G_{12}^{\prime}, \zeta=-\eta^{2}$, and $q=\eta^{3}$. Hence $\mathcal{D}=\mathcal{D}_{92}$.

Case 2bbb2b2. If $-q^{2}=\zeta$, then $q \in G_{12}^{\prime}$ and $p \neq 2$ since $q \neq \zeta^{-1}$. Hence $\mathcal{D}=\mathcal{D}_{81}$.

Case 2bbb3. If the condition (b3) holds, then $\left(-\zeta q^{2}\right)^{3}(\zeta q)^{-1}=1$ or $\sum_{i=0}^{3}\left(-\zeta q^{2}\right)^{i}=0,1-\zeta q^{2} \neq 0$.

Case 2bbb3a. Consider the equation $\left(-\zeta q^{2}\right)^{3}(\zeta q)^{-1}=1$, that is $-q^{5}=\zeta$. Hence $q=-\zeta^{-1}$ or $-q \in G_{15}^{\prime}, p \neq 5$. 
Case 2bbb3a1. If $-q \in G_{15}^{\prime}, p \neq 3,5$, and $\zeta=-q^{5}$, then $\mathcal{D}=\mathcal{D}_{16,2}$.

Case 2bbb3a2. If $q=-\zeta^{-1}$ then $p \neq 2$ since $q \neq \zeta^{-1}$. Hence the Dynkin diagrams of $r_{1}(X), X$ and $r_{2}(X)$, respectively, are

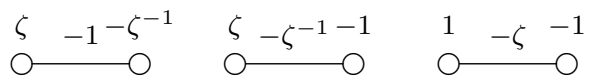

with $\zeta \in G_{3}^{\prime}$. Then one obtains that $a_{12}^{r_{2}(X)}=1-p$. We distinguish four cases.

Case 2bbb3a2a. If $p=5$, then $\mathcal{D}=\mathcal{D}_{16^{\prime \prime 2}}$.

Case 2bbb3a2b. If $p=7$, then $\mathcal{D}=\mathcal{D}_{18,2}$.

Case 2bbb3a2c. If $p=6 s+1(s \geq 2)$, then the Dynkin diagrams of $r_{1}(X), X, r_{2}(X), r_{1} r_{2}(X)$, $r_{2} r_{1} r_{2}(X)$, and $\left(r_{1} r_{2}\right)^{2}(X)$, respectively, are

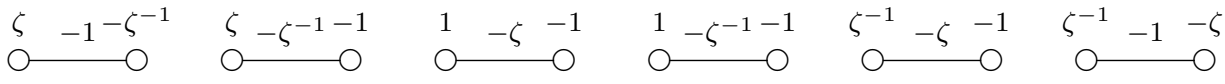

with $\zeta \in G_{3}^{\prime}$. Hence $n=6$ in Theorem 4.15 and $\left(c_{k}\right)_{k \geq 0}=(2,3,2,1, p-1,1,2,3,2,1, p-1,1)^{\infty}$. Then $l=20-2 p<0$, which is a contradiction to Theorem 4.15.

Case 2bbb3a2d. If $p=6 s+5$, where $s \geq 1$, then the Dynkin diagrams of $r_{1}(X), X, r_{2}(X)$ and $r_{1} r_{2}(X)$, respectively, are

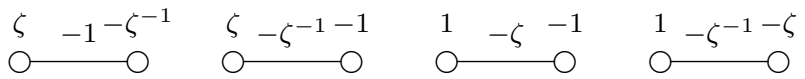

with $\zeta \in G_{3}^{\prime}$. Then $n=4$ and $\left(c_{k}\right)_{k \geq 0}=(2,3,2,1, p-1,1, p-1,1)^{\infty}$. Hence $l=16-2 p<0$. Again, one gets a contradiction.

Case 2bbb3b. Consider the equation $0=\sum_{i=0}^{3}\left(-\zeta q^{2}\right)^{i}=\left(1-\zeta q^{2}\right)\left(1+\zeta^{2} q^{4}\right)$, where $\zeta q^{2} \neq 1$. One gets $\zeta=-q^{4}$. If $p=2$, then $\zeta^{4}=q^{4}$ and hence $\zeta=q$, which is a contradiction to $\zeta q^{2} \neq 1$. Otherwise $q \in G_{24}^{\prime}$ and $\mathcal{D}=\mathcal{D}_{13,2}$.

Step 3. Now we change the label. It means that $\left(c_{k}\right)_{k \geq 1}$ is the characteristic sequence of $\mathcal{C}_{s}(M)$ with respect to $X$ and the label 2.

Assume that $\left(c_{0}, c_{1}, c_{2}\right)=\left(2,1, b^{\prime}\right)$, where $b^{\prime} \in\{3,4,5\}$. Then we obtain that $a_{12}^{X}=-2, a_{21}^{X}=$ -1 and $a_{12}^{r_{2}(X)}=-b^{\prime}$. If $q_{11}^{2} q_{0}=1$ and $q_{22}=-1$ then $a_{12}^{r_{2}(X)}=-2$, which is a contradiction. If $q_{0} q_{22}=1$ then $a_{12}^{r_{2}(X)}=a_{12}^{X}=-2$, which is again a contradiction. Suppose now that $1+q_{11}+q_{11}^{2}=0, q_{22}=-1$ and $q_{0} \notin\left\{1,-1, q_{11}^{-2}\right\}$. Since $a_{12}^{X}=-2$, we also obtain that $q_{0} \neq q_{11}^{-1}$.

Case 3a. If $p=3$, then by setting $q:=q_{0}$, the Dynkin diagrams of $X$ and $r_{2}(X)$, respectively, are

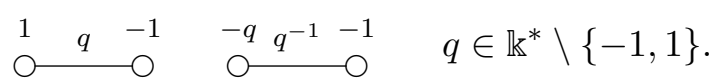

Case 3a1. If $p=3$ and $b^{\prime}=3$, then one gets $(-q)^{3} q^{-1}=1$ or $\sum_{i=0}^{3}(-q)^{i}=0$. Since $q \neq 1$, both equations imply that $q^{2}=-1$. Hence $\mathcal{D}=\mathcal{D}_{9^{\prime}, 2}$.

Case 3a2. If $p=3$ and $b^{\prime}=4$, then one has $(-q)^{4} q^{-1}=1$ or $\sum_{i=0}^{4}(-q)^{i}=0$. If $(-q)^{4} q^{-1}=1$ then $q=1$, which is a contradiction to (5.1). If $\sum_{i=0}^{4}(-q)^{i}=0$ then $q^{5}=-1$ and $\mathcal{D}=\mathcal{D}_{16^{\prime}, 2}$.

Case 3a3. If $p=3$ and $b^{\prime}=5$, then one obtains $(-q)^{5} q^{-1}=1$ or $\sum_{i=0}^{5}(-q)^{i}=0$. If $(-q)^{5} q^{-1}=1$ then $q \in G_{8}^{\prime}$ and $\mathcal{D}=\mathcal{D}_{13^{\prime}, 2}$. Consider the equation $0=\sum_{i=0}^{5}(-q)^{i}=(1-q)\left(1+q^{2}+q^{4}\right)$. Then $q^{2}=1$ since $p=3$ and $q \neq 1$, which is a contradiction to (5.1). 
Case $3 \mathrm{~b}$. We now consider the cases in which the condition $p \neq 3$ holds. Set $\zeta:=q_{11}$ and $q:=q_{0}$. The Dynkin diagram of $r_{2}(X)$ is

$\underset{-\zeta q}{-\zeta q^{-1}} \stackrel{-1}{\circ} \quad \zeta \in G_{3}^{\prime}, \quad q \in \mathbb{R}^{*} \backslash\left\{1,-1, \zeta, \zeta^{-1}\right\}$

Case 3 b1. If $p \neq 3$ and $b^{\prime}=3$, then one gets $(-\zeta q)^{3} q^{-1}=1$ or $\sum_{i=0}^{3}(-\zeta q)^{i}=0$. If $(-\zeta q)^{3} q^{-1}=1$ then $q \in G_{4}^{\prime}, p \neq 2$ and $\mathcal{D}=\mathcal{D}_{92}$. If $0=\sum_{i=0}^{3}(-\zeta q)^{i}=(1-\zeta q)\left(1+(\zeta q)^{2}\right)$, then $(\zeta q)^{2}=-1$ and $p \neq 2$ since $q \neq \zeta^{-1}$. Hence $\mathcal{D}=\mathcal{D}_{81}$.

Case $3 \mathrm{~b} 2$. If $p \neq 3$ and $b^{\prime}=4$, then one gets $(-\zeta q)^{4} q^{-1}=1$ or $\sum_{i=0}^{4}(-\zeta q)^{i}=0$. If $(-\zeta q)^{4} q^{-1}=1$ then $\zeta=q^{-3}$. Since $q \notin G_{3}^{\prime}$, one obtains $q \in G_{9}^{\prime}$ and $\mathcal{D}=\mathcal{D}_{10,2}$. The equation $\sum_{i=0}^{4}(-\zeta q)^{i}=0$ gives $\zeta=-q^{5}$. Since $\zeta \in G_{3}^{\prime}$, one gets $-q \in G_{3}^{\prime}, \zeta=-q^{-1}, p=5$ or $-q \in G_{15}^{\prime}, p \neq 3,5$. If $-q \in G_{3}^{\prime}$ then $\mathcal{D}=\mathcal{D}_{16^{\prime \prime}, 2}$ and if $-q \in G_{15}^{\prime}$ then $\mathcal{D}=\mathcal{D}_{16,2}$.

Case 3b3. If $p \neq 3$ and $b^{\prime}=5$, then one gets $(-\zeta q)^{5} q^{-1}=1$ or $\sum_{i=0}^{5}(-\zeta q)^{i}=0,-\zeta q \neq 1$. If $(-\zeta q)^{5} q^{-1}=1$ and $p=2$ then $q \zeta^{-1}=1$, which is a contradiction to $(5.2)$. If $(-\zeta q)^{5} q^{-1}=1$ and $p \neq 2$ then $\zeta=-q^{4}$ and $\mathcal{D}=\mathcal{D}_{13,2}$. Consider $0=\sum_{i=0}^{5}(-\zeta q)^{i}=(1-\zeta q)\left(1+(-\zeta q)^{2}+(-\zeta q)^{4}\right)$. Since $q \notin\left\{1,-1, \zeta, \zeta^{-1}\right\}$, one gets $p \neq 2$ and $q^{3}=-1$. Since $-\zeta q \neq 1$, one gets $q=-\zeta$ and $a_{12}^{r_{2}(X)}=-2$, which is a contradiction.

Step 4. Again we use the same labeling as in steps 1 and 2. Assume that $\left(c_{0}, c_{1}, c_{2}, c_{3}\right)=$ $\left(1,3,1, c^{\prime}\right)$, where $c^{\prime} \in\{3,4,5\}$. Then $a_{21}^{X}=-1$ and $a_{12}^{X}=-3$. We distinguish four cases: 4aa, $4 \mathrm{ab}, 4 \mathrm{ba}$ and $4 \mathrm{bb}$.

Case 4aa. If $q_{11}^{3} q_{0}=1$ and $q_{22} q_{0}=1$, then $\mathcal{D}=\mathcal{D}_{11,1}$.

Case 4 ab. Set $q:=q_{11}$. If $q_{11}^{3} q_{0}=1$ and $q_{22}=-1$, then the Dynkin diagrams of $X, r_{1}(X)$ and $r_{2} r_{1}(X)$, respectively, are

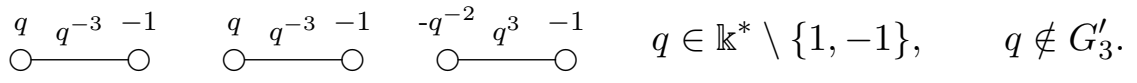

Then $-a_{12}^{r_{2} r_{1}(X)}=c^{\prime} \in\{3,4,5\}$. Hence we distinguish three cases: 4ab1, 4ab2 and 4ab3.

Case 4ab1. If $c^{\prime}=3$, then $\left(-q^{-2}\right)^{3} q^{3}=1$ or $\sum_{i=0}^{3}\left(-q^{-2}\right)^{i}=0,1-q^{-2} \neq 0$. If $\left(-q^{-2}\right)^{3} q^{3}=1$ then $\mathcal{D}=\mathcal{D}_{11,1}$, where $q^{3}=-1$. If $\sum_{i=0}^{3}\left(-q^{-2}\right)^{i}=0$, then $\mathcal{D}=\mathcal{D}_{12,1}$.

Case $4 \mathrm{ab} 2$. If $c^{\prime}=4$, then $\left(-q^{-2}\right)^{4} q^{3}=1$ or $\sum_{i=0}^{4}\left(-q^{-2}\right)^{i}=0$.

Case 4ab2a. The equation $\left(-q^{-2}\right)^{4} q^{3}=1$ gives $q^{5}=1$ and $p \neq 5$, since $q \neq 1$. Hence $\mathcal{D}=\mathcal{D}_{14,1}$.

Case $4 \mathrm{ab} 2 \mathrm{~b}$. Consider the equation $\sum_{i=0}^{4}\left(-q^{-2}\right)^{i}=0$. One gets $q^{10}=-1$. If $p=2$ then $\mathcal{D}=\mathcal{D}_{14,1}$. If $p=5$ then $q^{2}=-1$ and $\mathcal{D}=\mathcal{D}_{15^{\prime}, 1}$. If $p \neq 2,5$, then $q \in G_{20}^{\prime}$ and $\mathcal{D}=\mathcal{D}_{15,1}$.

Case 4ab3. If $c^{\prime}=5$, then $\left(-q^{-2}\right)^{5} q^{3}=1$ or $\sum_{i=0}^{5}\left(-q^{-2}\right)^{i}=0$.

Case 4ab3a. Consider the equation $\left(-q^{-2}\right)^{5} q^{3}=1$, which gives $-q^{7}=1$. Since $q \neq-1$, one gets $p \neq 7$ and $-q \in G_{7}^{\prime}$. Hence $\mathcal{D}=\mathcal{D}_{17,1}$. 
Case 4ab3b. Consider the equation $0=\sum_{i=0}^{5}\left(-q^{-2}\right)^{i}=\left(1-q^{-2}\right)\left(1+q^{-4}+q^{-8}\right)$. Since $q^{2} \neq 1$, one gets $1+q^{-4}+q^{-8}=0$. If $p=3$ then $q \in G_{4}^{\prime}$ since $q^{2} \neq 1$. Hence $a_{12}^{r_{2} r_{1}(X)}=-2$, which is a contradiction. Then $p \neq 3$ and $q^{-4} \in G_{3}^{\prime}$. Since $c^{\prime}=5$, one gets $q \in G_{6}^{\prime}$ or $q \in G_{12}^{\prime}$. If $q \in G_{6}^{\prime}$, then $a_{12}^{r_{2} r_{1}(X)}=-3$, which is a contradiction. If $q \in G_{12}^{\prime}$, then $a_{12}^{r_{2} r_{1}(X)}=-2$, which is again a contradiction.

Case 4ba. The conditions $1+q_{11}+q_{11}{ }^{2}+q_{11}^{3}=0, q_{11} \neq-1$ and $q_{22} q_{0}=1$ hold. Then $q_{11} \in G_{4}^{\prime}$ and $p \neq 2$ since $a_{12}^{X}=-3$. Set $\zeta:=q_{11}$ and $q=q_{22}$. The Dynkin diagram of $r_{1}(X)$ is $\quad \zeta \quad-q q^{-2}$ with $\zeta \in G_{4}^{\prime}$ and $q \in \mathbb{k}^{*} \backslash\left\{1,-1, \zeta, \zeta^{-1}\right\}$. Since $-a_{21}^{r_{1}(X)}=c_{2}=1$, one gets $\left(\zeta q^{-2}\right)(-q)=1$ or $\zeta q^{-2}=-1$. If $\left(\zeta q^{-2}\right)(-q)=1$ then $q=-\zeta$, which is a contradiction. If $\zeta q^{-2}=-1$ then $q \in G_{8}^{\prime}$ and $\mathcal{D}=\mathcal{D}_{12,3}$.

Case 4bb. Consider the last case: $1+q_{11}+q_{11}^{2}+q_{11}^{3}=0, q_{22}=-1$ and $q_{11} \neq-1$. Then $q_{11}^{2}=-1$ and $p \neq 2$. Set $\zeta:=q_{11}$ and $q=q_{0}$. The Dynkin diagram of $r_{1}(X)$ is $\begin{gathered}\zeta-q^{-1-\zeta q^{3}} \text { with } \\ \mathrm{O}\end{gathered}$ $q \in \mathbb{k}^{*} \backslash\left\{1,-1, \zeta, \zeta^{-1}\right\}$ and $\zeta \in G_{4}^{\prime}$. Since $-a_{21}^{r_{1}(X)}=c_{2}=1$, one has $\left(-q^{-1}\right)\left(-\zeta q^{3}\right)=1$ or $\zeta q^{3}=1$.

Case 4bb1. If $\zeta q^{3}=1$, then $\zeta=q^{-3} \in G_{4}^{\prime}$. If $p=3$, then $\zeta=q$, which is a contradiction. Hence $p \neq 3$ and $\mathcal{D}=\tau \mathcal{D}_{85}$.

Case 4 bb2. The condition $\left(-q^{-1}\right)\left(-\zeta q^{3}\right)=1$ holds. Then $\zeta=q^{-2} \in G_{4}^{\prime}$. Hence $q \in G_{8}^{\prime}$ and $\mathcal{D}=\mathcal{D}_{12,2}$.

By checking all cases in Theorem 4.6, the proof of Theorem 5.1 is completed.

Remark 5.3. Assume that char $\mathbb{k}=p>0$. Let $V$ be a two-dimensional braided vector space of diagonal type. Let $\left(x_{1}, x_{2}\right)$ be a basis of $V$ and $\left(q_{i j}\right)_{1 \leq i, j \leq 2} \in(\mathbb{k} \backslash\{0\})^{2 \times 2}$ satisfying

$$
c\left(x_{i} \otimes x_{j}\right)=q_{i j} x_{j} \otimes x_{i}
$$

for any $i, j$.

- By [13, Corollary 6], $\operatorname{dim}_{\mathbb{k}} \mathcal{B}(V)<\infty$ if and only if $M=\left(\mathbb{k} x_{1}, \mathbb{k} x_{2}\right)$ admits all reflections, $\mathcal{W}(M)$ is finite, and for all points $\mathcal{D}$ of $\mathcal{C}_{s}(M)$, the labels of the vertices of $\mathcal{D}$ are roots of unity (including 1 ). Therefore with Theorem 5.1 one can easily decide whether $\operatorname{dim}_{\mathbb{k}} \mathcal{B}(V)$ is finite.

- If the Dynkin diagram of $V$ appears in the row 18 of Table 5.6, then the Weyl groupoid of $\mathcal{B}(V)$ is not appearing for Nichols algebras in characteristic zero.

\section{Acknowledgements}

It is a pleasure to thank N. Andruskiewitsch and H.-J. Schneider for a very fruitful discussion on some details of this topic. The authors thank the referees for their helpful comments and suggestions. J. Wang is supported by China Scholarship Council.

\section{References}

[1] Andruskiewitsch N., About finite dimensional Hopf algebras, in Quantum Symmetries in Theoretical Physics and Mathematics (Bariloche, 2000), Contemp. Math., Vol. 294, Amer. Math. Soc., Providence, RI, 2002, $1-57$.

[2] Andruskiewitsch N., Graña M., Braided Hopf algebras over non-abelian finite groups, Bol. Acad. Nac. Cienc. (Córdoba) 63 (1999), 45-78, math.QA/9802074.

[3] Andruskiewitsch N., Heckenberger I., Schneider H.-J., The Nichols algebra of a semisimple Yetter-Drinfeld module, Amer. J. Math. 132 (2010), 1493-1547, arXiv:0803.2430. 
[4] Andruskiewitsch N., Schneider H.-J., Lifting of quantum linear spaces and pointed Hopf algebras of order $p^{3}$, J. Algebra 209 (1998), 658-691, math.QA/9803058.

[5] Andruskiewitsch N., Schneider H.-J., Finite quantum groups and Cartan matrices, Adv. Math. 154 (2000), $1-45$.

[6] Andruskiewitsch N., Schneider H.-J., Pointed Hopf algebras, in New Directions in Hopf Algebras, Math. Sci. Res. Inst. Publ., Vol. 43, Cambridge University Press, Cambridge, 2002, 1-68, math.QA/0110136.

[7] Andruskiewitsch N., Schneider H.-J., On the classification of finite-dimensional pointed Hopf algebras, Ann. of Math. 171 (2010), 375-417, math.QA/0502157.

[8] Cibils C., Lauve A., Witherspoon S., Hopf quivers and Nichols algebras in positive characteristic, Proc. Amer. Math. Soc. 137 (2009), 4029-4041, arXiv:0901.4408.

[9] Cuntz M., Heckenberger I., Weyl groupoids of rank two and continued fractions, Algebra Number Theory 3 (2009), 317-340, arXiv:0807.0124.

[10] Cuntz M., Heckenberger I., Weyl groupoids with at most three objects, J. Pure Appl. Algebra 213 (2009), 1112-1128, arXiv:0805.1810.

[11] Cuntz M., Heckenberger I., Reflection groupoids of rank two and cluster algebras of type A, J. Combin. Theory Ser. A 118 (2011), 1350-1363, arXiv:0911.3051.

[12] Heckenberger I., The Weyl groupoid of a Nichols algebra of diagonal type, Invent. Math. 164 (2006), 175188.

[13] Heckenberger I., Rank 2 Nichols algebras with finite arithmetic root system, Algebr. Represent. Theory 11 (2008), 115-132, math.QA/0412458.

[14] Heckenberger I., Classification of arithmetic root systems, Adv. Math. 220 (2009), 59-124, math.QA/0605795.

[15] Heckenberger I., Schneider H.-J., Nichols algebras over groups with finite root system of rank two I, J. Algebra 324 (2010), 3090-3114.

[16] Heckenberger I., Schneider H.-J., Root systems and Weyl groupoids for Nichols algebras, Proc. Lond. Math. Soc. 101 (2010), 623-654, arXiv:0807.0691.

[17] Heckenberger I., Schneider H.-J., Right coideal subalgebras of Nichols algebras and the Duflo order on the Weyl groupoid, Israel J. Math. 197 (2013), 139-187, arXiv:0909.0293.

[18] Heckenberger I., Yamane H., A generalization of Coxeter groups, root systems, and Matsumoto's theorem, Math. Z. 259 (2008), 255-276, math.QA/0610823.

[19] Kac V.G., Infinite-dimensional Lie algebras, 3rd ed., Cambridge University Press, Cambridge, 1990.

[20] Kharchenko V.K., A quantum analogue of the Poincaré-Birkhoff-Witt theorem, Algebra and Logic 38 (1999), 259-276, math.QA/0005101.

[21] Lusztig G., Introduction to quantum groups, Modern Birkhäuser Classics, Birkhäuser/Springer, New York, 2010.

[22] Nichols W.D., Bialgebras of type one, Comm. Algebra 6 (1978), 1521-1552.

[23] Rosso M., Quantum groups and quantum shuffles, Invent. Math. 133 (1998), 399-416.

[24] Schauenburg P., A characterization of the Borel-like subalgebras of quantum enveloping algebras, Comm. Algebra 24 (1996), 2811-2823.

[25] Semikhatov A.M., Virasoro central charges for Nichols algebras, arXiv:1109.1767.

[26] Semikhatov A.M., Tipunin I.Yu., The Nichols algebra of screenings, Commun. Contemp. Math. 14 (2012), 1250029, 66 pages, arXiv:1101.5810.

[27] Semikhatov A.M., Tipunin I.Yu., Logarithmic $\widehat{s \ell(2) ~ C F T ~ m o d e l s ~ f r o m ~ N i c h o l s ~ a l g e b r a s: ~ I, ~ J . ~ P h y s . ~ A: ~ M a t h . ~}$ Theor. 46 (2013), 494011, 53 pages, arXiv:1301.2235.

[28] Woronowicz S.L., Compact matrix pseudogroups, Comm. Math. Phys. 111 (1987), 613-665.

[29] Woronowicz S.L., Differential calculus on compact matrix pseudogroups (quantum groups), Comm. Math. Phys. 122 (1989), 125-170. 